Themenheft Nr. 46: Eltern - Pädagog*innen - Medienkompetenzen.

Eltern und Pädagog*innen zwischen Aneignung und Vermittlung von Medienkompetenz. Herausgegeben von Thorsten Naab, Alexandra Langmeyer, Ruth Wendt und Jessica Kühn

\title{
Vom Reizthema zum Qualitätsmerkmal - Die Coronapandemie als Gamechanger frühkindlicher Medienerziehung?
}

\section{Eine Analyse neuer Potenziale der pandemiebedingten Digitalisierungs- prozesse in Kindertageseinrichtungen}

\author{
Theresa Lienau $^{1}$ (D) und Elena Frense ${ }^{2}$ (D) \\ ${ }^{1}$ Stiftung Digitale Chancen \\ ${ }^{2}$ Der Kinderschutzbund Bundesverband
}

\begin{abstract}
Zusammenfassung
Während des ersten Covid-19-bedingten Lockdowns wurden die Rufe nach einer umfassenden Digitalisierung von Bildungsinstitutionen laut. Die Vermittlung von dafür notwendigen frühkindlichen Medienkompetenzen ist jedoch noch lange nicht im Alltag von Kitas angekommen. Es stellt sich die Frage, inwieweit die Extremsituation, in der sich Kitas und Familien während des ersten Lockdowns befanden, einen Gamechanger darstellt: Konnten die ausgelösten Digitalisierungsprozesse Eltern und Fachkräfte für die Potenziale digitaler Medien in der Kita sensibilisieren? Und inwieweit konnten Fachkräfte im Umgang mit digitalen Medien Hemmungen abbauen und Kompetenzen stärken? In der vorgestellten mehrstufigen Mixed-Methods-Studie wurden zwischen März und Dezember 2020 in zehn Kindertagesstätten sowohl Eltern als auch Fachkräfte befragt. Die Ergebnisse zeigen, dass es durch den ersten Lockdown in den befragten Kitas diverse Anstösse hin zu einer offeneren und positiveren Grundhaltung gegenüber digitalen Medien gab. Etablierte analoge Praktiken wurden in vielen Bereichen aufgebrochen oder überdacht und stärker digitalisiert. Seitens der Eltern lassen sich - trotz einer Zunahme der kindlichen Mediennutzung im ersten Lockdown - keine wesentlichen Haltungsveränderungen hinsichtlich der Notwendigkeit einer gezielten Medienerziehung beobachten. Die Ergebnisse deuten darauf hin, dass die positiven Erfahrungen, die Kitas mit digitalen Medien gemacht haben, ein notwendiger erster Schritt zu einer nachhaltigen Verankerung von Medienpädagogik, jedoch keineswegs ein ‘Selbstläufer) sind.
\end{abstract}


From a Sensitive Topic to a Quality Feature - The Corona Pandemic as a Gamechanger for Early Childhood Media Education? An Analysis of new Potentials of the Pandemic-Related Digitization Processes in German Child Day Care Facilities

\begin{abstract}
During the first Covid-19-related lockdown in Germany, demands for the comprehensive digitization of educational institutions got louder. However, media education in early childcare institutions is still hardly integrated into daily practices of daycare centers. The question arises to what extent the extreme situation in which daycare centers and families found themselves during the first lockdown was a gamechanger: Were the triggered digitization processes able to raise awareness among parents and professionals for the potentials of digital media in daycare institutions? And to what extent were professionals able to reduce their inhibitions and strengthen their competencies in dealing with digital media? In the present mixed-methods study composed of semi-structured interviews and a quantitative questionnaire, parents and professionals of ten daycare centers were surveyed and interviewed between March and December 2020. The results show that the first lockdown provided various impulses for a more open and positive attitude towards digital media. Established non-digital practices were broken up or reconsidered in many areas. Despite the increase in children's use of media in the first lockdown, no significant changes in attitude regarding the need for media education was observed on the part of parents. The results suggest that the positive experiences that daycare centers had with digital media are a necessary first step towards a more sustainably anchored media education - but by no means a foregone conclusion.
\end{abstract}

\title{
1. Einleitung
}

In der Zeit des ersten Covid-19-bedingten Lockdowns wurden die Rufe nach einer umfassenden Digitalisierung von Bildungsinstitutionen laut. Medienkompetenz stellt die Basis dieser Digitalisierungsprozesse dar - ihre Vermittlung auch in der frühkindlichen Bildung wird bereits seit Jahrzehnten gefordert (etwa Six u. a. 1989). Kinder und Jugendliche sollen zu einem souveränen Umgang mit digitalen Medien befähigt und ermutigt werden, sich als aktive Gestaltende der digitalen Welt denn als passive Rezipierende begreifen. Doch die Vermittlung von Medienkompetenz(en) ist noch lange nicht im Alltag von Kitas angekommen. Eine Umfrage der Stiftung Haus der kleinen Forscher (2017) ergab, dass nicht einmal in jeder fünften Kita der Einsatz und Umgang mit digitalen Medien im Konzept geregelt ist (17). Eine Untersuchung im Rahmen der sogenannten MoFam-Studien besagt, dass in den meisten Einrichtungen digitale Medien im pädagogischen Alltag nur eine kleine Rolle spielen und andere Erziehungsbereiche vielfach als relevanter angesehen werden (Schubert u. a. 2018b, 21). 
Die Coronapandemie könnte hier eine Zäsur darstellen: Das breite Thema der Digitalisierung hat während der Coronakrise besondere Aufmerksamkeit im gesellschaftlichen Diskurs erfahren. Mit Blick auf die frühkindliche Bildung wurde aufgezeigt, dass digitale Medien insbesondere während der lockdownbedingten Schliessungen der Kitas diverse Möglichkeiten bieten, um ihrem Bildungsauftrag weiterhin nachzukommen (vgl. BMFSFJ 2020). Ein in den Medien häufig verwendetes Beispiel dafür war der «digitale Morgenkreis» (u. a. Bartels 2020; Damm 2020; Eckert 2020; Grüling 2020; Heundorf 2020). Der Fachverband Katholischer Tageseinrichtungen für Kinder (KTK) fordert gar einen Digital-Pakt für Kitas, wie der Vorsitzende Clemens Bieber ausführt: «Vor allem in den ersten Wochen der Coronapandemie wurde in unseren Kitas deutlich, wie wichtig eine digitale Ausstattung für die pädagogische Arbeit ist. Ein Grossteil der pädagogischen Fachkräfte musste auf private digitale Medien zurückgreifen, um die Kontaktaufnahme mit den Kindern zu Hause zu gewährleisten.» (Deutscher Caritasverband 2020). Diese Forderung stützte auch die damalige Familienministerin Franziska Giffey (BMFSFJ 2021). Angesichts des üblicherweise geringen Stellenwertes von digitalen Medien im Alltag von Kitas ist das öffentliche Interesse an dieser Thematik durchaus als neuartig zu beschreiben.

Unabhängig von der Covid-19-Pandemie werden in der Literatur immer wieder zwei Faktoren genannt, die die Digitalisierung in Kindertagesstätten bremsen. So wird regelmässig auf die mangelnden Medien- und medienpädagogischen Kompetenzen von Fachkräften verwiesen und entsprechend ihr Bedarf an Fort-, Aus- und Weiterbildung abgeleitet (vgl. Six u. a. 1998; Six und Gimmler 2007; FriedrichsLiesenkötter 2016; Eder u. a. 2013). Ebenso wird auf die ablehnenden Haltungen von Kita-Leitungen und Fachkräften (vgl. Schubert u. a. 2018a; Anfang und Demmler 2018; Friedrichs-Liesenkötter 2016, 2018; Meister u. a. 2012; Six u. a. 1998; Six und Gimmler 2007) wie auch Eltern (Fleischer u. a. 2018; Schubert u. a. 2018a; Institut für Demoskopie Allensbach 2014; Theunert und Demmler 2006) verwiesen. Vor dem Hintergrund der Entwicklungen im Kontext des ersten Covid-19-bedingten Lockdowns stellt sich die Frage, inwieweit die Extremsituation, in der sich Kindertagesstätten und Familien befanden, einen Gamechanger in Bezug auf diese Aspekte darstellt. Konnten die pandemiebedingt ausgelösten Digitalisierungsprozesse - explizit im ersten Lockdown - Eltern und Fachkräfte für die Relevanz und Potenziale digitaler Medien in der Kita sensibilisieren? Aus dieser Kernfrage ergeben sich die folgenden beiden forschungsleitenden Fragen, die sich auf jeweils eine der beiden Gruppen beziehen:

1. Inwieweit konnten Fachkräfte durch den verstärkten Umgang mit digitalen Medien Hemmungen abbauen und digitale Kompetenzen stärken?

2. Inwieweit wurde durch eine verstärkte häusliche Mediennutzung das Bewusstsein der Eltern für die Bedeutung von Medien im Alltag von Kindern und eine entsprechende medienerzieherische Begleitung gestärkt? 
Diese beiden forschungsleitenden Fragen beziehen sich auf Haltungsänderungen zweier unterschiedlich grosser und heterogener gesellschaftlicher Gruppen: die der Eltern und die der pädagogischen Fachkräfte in Kitas. Das Ziel der hier vorgestellten Studie kann (aufgrund der hohen Aktualität des Themas sowie der Studie) keine abschliessende Beantwortung der Fragestellung sein - vielmehr wurde mittels eines mehrstufigen Forschungsdesigns mit Fachkräften und Eltern aus zehn Kindertageseinrichtungen untersucht, inwiefern sich Anhaltspunkte für die formulierten forschungsleitenden Fragen erkennen lassen.

Da sich die vorliegende Studie neben den (technischen) Entwicklungen in den Kindertageseinrichtungen insbesondere für Haltungsveränderungen von pädagogischen Fachkräften und Eltern interessiert, steht der Diskurs über die frühkindliche Medienerziehung und damit verbundene Aushandlungsprozesse im Zentrum der Untersuchung. Um nachzuvollziehen, welchen Einfluss die Coronapandemie auf die Haltungen von Erzieherinnen, Erziehern und Eltern gegenüber der frühkindlichen Medienerziehung haben könnte, wird zunächst auf mögliche fachkräfte- bzw. kitabezogene Entwicklungen und anschliessend auf die Perspektive der Eltern eingegangen. Nach einem ersten Überblick über den aktuellen Forschungsstand, werden sowohl für die Seite der Erzieherinnen und Erziehern als auch für die Seite der Eltern Annahmen bezüglich möglicher Haltungsänderungen formuliert, die es zu überprüfen gilt.

Für ein genaueres Verständnis des Diskurses zur frühkindlichen Medienerziehung sei an dieser Stelle auf eine begriffliche Unterscheidung hingewiesen. In dieser Studie werden die Begriffe Medienerziehung bzw. -pädagogik verwendet. Medienpädagogik kann als Überbegriff für «alle pädagogisch orientierten Beschäftigungen mit Medien in Theorie und Praxis» (Baacke 2007, 7) verstanden werden. Da wir uns nicht nur auf pädagogische bzw. erzieherische Handlungen von Fachkräften, sondern auch von Eltern beziehen, stützen wir uns auf eine Definition des Begriffs Medienerziehung, die pädagogische Handlungen ausserhalb institutioneller Bildungseinrichtungen einbezieht. Tulodziecki (2008) zufolge umfasst der Begriff

«alle Aktivitäten und Überlegungen in Erziehung und Bildung [...], die das Ziel haben, ein humanes bzw. verantwortliches Handeln im Zusammenhang mit der Mediennutzung und Mediengestaltung zu entwickeln.» (ebd., 110).

Des Weiteren wird auf die Kompetenzen von Fachkräften im Umgang mit digitalen Medien Bezug genommen. Im Kontext dieser Studie steht die sichere Nutzung und Bedienung der Geräte im Vordergrund, während das Medienkompetenzmodell von Baacke (2007) darüber hinausgeht und verdeutlicht, dass eine tiefergehende Medienkompetenz auch kritisch-analytische, reflexive oder ethische Fähigkeiten umfasst (vgl. Rosebrock und Zitzelsberger 2002). 


\section{Forschungsstand}

Das Forschungsinteresse an den durch die Covid-19-Pandemie ausgelösten gesellschaftlichen Veränderungen ist enorm ${ }^{1}$, umfasst jedoch bislang fast ausschliesslich quantitative Erhebungen. Einige Studien nehmen dabei auch das seit dem Ausbruch der Coronapandemie veränderte Mediennutzungsverhalten von Kindern und Jugendlichen in den Blick und zeigen auf, dass durch die verschiedenen Altersstufen hinweg die Nutzung digitaler Medien zugenommen hat (Cohen u. a. 2020; Langmeyer 2020; Rathgeb 2020). Erste Daten zum Kontakt zwischen Kitas und Familien in der Covid-19-Pandemie erhoben Cohen u. a. (2020); die Forschungslandschaft ist in diesem Bereich jedoch noch sehr schwach ausgeprägt. Offen ist bislang, inwiefern die pandemiebedingten Entwicklungen auch mittel- und langfristig zu Veränderungen in der Breite der Kindertagesstätten führen und eine stärkere Verankerung von medienpädagogischen Inhalten im pädagogischen Alltag vorangetrieben wird.

Die Literatur zeigt - wie oben bereits kurz skizziert -, dass digitale Medien in Kindertageseinrichtungen bislang allenfalls sehr eingeschränkt eingesetzt werden. Six und Gimmler veröffentlichten zwei Studien (Six u. a. 1989; Six und Gimmler 2007), die zeigen, dass sich in den knapp zehn dazwischenliegenden Jahren kaum etwas hinsichtlich der Verankerung medienerzieherischer Inhalte sowohl in Kitas als auch der Erzieherinnen und -erzieherausbildung verändert hat (Six und Gimmler 2007, 273). Tiefergehend halten sie fest, dass die «theoretische Basis für ein engagiertes und erfolgreiches medienerzieherisches Handeln in den Kindergärten in vielen Fällen offenbar nach wie vor nicht gegeben [sei]», da die befragten Erzieherinnen und Erzieher wenn überhaupt ein nur floskelhaftes Verständnis davon haben, was unter dem Bildungsbereich Medienerziehung zu verstehen ist, wie dieser aussehen kann oder was damit erreicht werden soll - genau diesem Verständnis käme aber eine Schlüsselrolle zu (ebd., 283). Six und Gimmler (2007) beschreiben ebenfalls, dass die eigene Medienkompetenz eine Basis für die medienpädagogische Kompetenz der Erzieherinnen und Erzieher bildet (ebd., 30 ff.). Sie kommen zu dem Ergebnis: «Ihre Medienkompetenz schätzen sie [die befragten Erzieherinnen und Erzieher, Anm. d. Verf.] offenbar insgesamt nicht sehr positiv ein, wobei sie sich allerdings mit Blick auf das Fernsehen weitaus kompetenter fühlen als für den Umgang mit Computer oder Internet.» (ebd., 135) ${ }^{2}$.

1 Diverse Studien und Publikationen gehen auf den veränderten Alltag von Eltern und Familien (Andresen u. a. 2020a; Andresen u. a. 2020b; BMFSFJ 2020; Blom u. a. 2020; Bujard u. a. 2020, Entringer und Krieger 2020; Liebig 2020; Zinn 2020; Zinn u. a. 2020) sowie von Kindern und Jugendlichen (Anger u. a. 2020; GeisThöne 2020; Götz u. a. 2020; Hajok 2020; Huebener u. a. 2020) ein. Auch die Veränderungen der Betreuungssituation in Kitas werden in der wöchentlich aktualisierten Corona-Kita-Studie (DJI und RKI 2020) sehr genau beobachtet.

2 Ähnlich auch bei Schubert u. a. 2018a, hier berichten befragte Fachkräfte, dass sie weniger kompetent im Umgang mit digitalen Medien seien als die Kinder; diese «hätten weniger Angst, etwas falsch zu machen» (15). 
Auch nutzten sie zum Untersuchungszeitpunkt wesentlich weniger Medien als der Durchschnitt der erwachsenen Bevölkerung (ebd., 129 ff.).

Es existieren bislang keine Studien, die seit Six und Gimmler (2007) einen wesentlichen Sprung in der Verankerung von Medienpädagogik in Kitas beobachtet haben. Zwar zeigen Lienau und van Roessel (2019b), dass seit 2012 in den Bildungs- und Erziehungsplänen für den frühkindlichen Bildungsbereich das Thema Medienerziehung mehr Aufmerksamkeit erfährt, jedoch weiterhin sehr viel knapper als andere Bildungsbereiche behandelt wird. Darüber hinaus ist es zweifelhaft, inwiefern die Vorgaben der Bildungspläne eine direkte Umsetzung in den Kitas erfahren, da es mit Ausnahme des Landes Berlin kein Bundesland gibt, dass die Implementierung bislang flächendeckend überprüft und begleitet (Lienau und van Roessel 2019b). Die zunehmende Verankerung in den Bildungsplänen muss daher nicht mit einer zunehmenden medienpädagogischen Praxis in den Kitas einhergehen. Schubert u. a. (2018b) zeigen, dass in etwa einem Drittel der 33 befragten bayerischen Kitas digitale Medien in der pädagogischen Arbeit mit Kindern genutzt werden, in der Mehrheit jedoch kaum eine Rolle spielen und von vielen Kitas als wenig relevant empfunden werden. Es hängt entsprechend vom persönlichen Engagement und Interesse der Fachkräfte und Leitungen ab, inwieweit Medienerziehung in der Kita umgesetzt wird (Schubert u. a. 2018a).

Friedrichs-Liesenkötter (2016) zeigt, dass bereits in der Ausbildung eine ablehnende Haltung gegenüber digitalen Medien unter angehenden pädagogischen Fachkräften weit verbreitet ist und diese häufig bewahrpädagogische Haltungen vertreten (ebd., 225; ebd., 353). Der mediale Habitus der Erzieherinnen und Erzieher habe zudem einen wesentlichen Einfluss auf ihren medienerzieherischen Habitus (u. a. ebd., $327 \mathrm{ff}$.). Im Rahmen der Studie ebenfalls befragte Lehrkräfte in der erzieherischen Ausbildung sagen aus, dass die Auszubildenden zwar meist eine hohe Mediennutzung aufwiesen, diese jedoch wenig reflektiert oder kritisch sei (ebd., 202). Um die Auszubildenden für die Relevanz des Themas Medienerziehung zu sensibilisieren, seien v.a. medienpädagogische Praxiserfahrungen in den Einrichtungen wichtig; diese blieben jedoch häufig aus. Zusammengefasst zeigen die zitierten Studien, dass digitale Medien in den meisten Kindertageseinrichtungen (und insbesondere im pädagogischen Bereich) nur sehr eingeschränkt eingesetzt werden. Hierfür sind oft ablehnende Haltungen des pädagogischen Fachpersonals ausschlaggebend. Diese können jedoch durch positive praktische Erfahrungen und die zunehmende Sicherheit im Umgang mit den Geräten verändert werden. Diese positiven Erfahrungen mit digitalen Medien werden jedoch im Kita-Alltag häufig nicht gefördert. Die Annahme, dass die Coronapandemie hier zu Veränderungen führen kann, äussert auch Grüling (2020) in einem journalistischen Artikel: 
«Ein positiver Nebeneffekt der Anstrengungen [des digitalen Kontakts zwischen Kita und Familien, Anm. d. Verf.] ist, dass viele Erzieherinnen [sic!] im Moment selbst viele neue Ideen ausprobieren. Davon könnte die digitale Bildung im Kindergarten immens profitieren - wenn irgendwann die Normalität in den Kita-Alltag zurückgekehrt ist.». (Grüling 2020)

Entsprechend lassen sich auf Basis des Forschungsstands für die Gruppe der Fachkräfte folgende forschungsleitende Annahmen konkretisieren, die es im Weiteren zu analysieren und überprüfen gilt:

- Annahme 1a: Kindertagesstätten haben während der Pandemie diverse Prozesse, die im normalen Alltag analog ablaufen, teilweise oder vollständig über digitale Wege realisiert.

- Annahme 1b: Pädagogische Fachkräfte haben durch die vermehrte Nutzung digitaler Medien ihre Kompetenzen im Umgang mit digitalen Geräten ausgebaut.

- Annahme 1c: Die grössere Sicherheit im Umgang mit digitalen Geräten hat bei pädagogischen Fachkräften zu einem Abbau von Vorbehalten hinsichtlich des Einsatzes digitaler Medien in ihrer (pädagogischen) Arbeit geführt.

In Bezug auf die Perspektiven von Eltern auf das Thema der kindlichen Mediennutzung und -erziehung zeigt der Forschungsstand, dass Familien mit kleinen Kindern einen sehr hohen Besitz an digitalen Endgeräten aufweisen (vgl. etwa Feierabend u. a. 2015). Auch nutzen Kinder digitale Medien - insbesondere solche, die durch Touchscreens leichter für sie zu bedienen sind (Eggert und Wagner 2016) - in immer jüngerem Alter und mit steigender Nutzungsdauer (vgl. Guth 2018; Feierabend u. a. 2015). Obwohl im privaten Bereich fast alle Kinder mit digitalen Medien in Kontakt kommen, sind viele Eltern gegenüber dem Einsatz in der Kita kritisch eingestellt (Grobbin 2016, 31 ff.; Feierabend u. a. 2015; Institut für Demoskopie Allensbach 2014). Theunert und Demmler (2006) bezeichnen den Mediengebrauch von kleinen Kindern als «pädagogisches Reizthema» (1). Schubert u. a. (2018a) beschreiben allerdings die Erfahrung von medienpädagogisch arbeitenden Kitas, dass Eltern auf konkrete Medienprojekte und deren Erläuterung durch die pädagogischen Fachkräfte durchaus positiv reagiert hätten (36 ff.; s. auch Lienau und van Roessel 2019a, 45 f.). Bei den Eltern ist also - analog zu den Erhebungen mit Erzieherinnen und Erziehern (s.o.) - davon auszugehen, dass Unklarheiten bestehen, was mit Medienerziehung gemeint ist (bzw. wie eine praktische Umsetzung aussieht) und dass diese Unsicherheit wesentlich ausschlaggebend für die kritischen Haltungen ist. Die als kritisch antizipierten Haltungen der Eltern werden von Fachkräften teilweise als Grund für die fehlende Umsetzung von Medienerziehung angebracht (Friedrichs-Liesenkötter 2016, 206; Lienau und van Roessel 2019a, 39). Auch Eder (2018) argumentiert, dass die medienpädagogische Arbeit in der Kita dadurch erschwert oder behindert werden könne, wenn Eltern diese für unnötig oder gar schädlich halten. 
Die hohe Bedeutung der Einbeziehung der Eltern in die medienpädagogische Arbeit der Kita wird in diversen Studien und Modellversuchen immer wieder hervorgehoben (siehe dazu genauer Lienau und van Roessel 2019a). Dennoch gestalte sich diese meist als schwierig bzw. werde nicht umgesetzt - hinderliche Faktoren seien dabei sowohl, dass Fachkräfte oftmals einen defizitären Blick auf die Mediennutzung der Eltern und Familien werfen, als auch, dass klassische Formate der Elternkooperation (wie etwa der Elternabend) nicht immer geeignet sind, um zu diesem Thema in den Austausch zu gehen (Schubert u. a. 2018a, 41).

Zusammenfassend lässt sich festhalten, dass mehrere Studien die Notwendigkeit hervorheben, Eltern stärker für das Thema Medienerziehung zu sensibilisieren. Insbesondere hinsichtlich des Einsatzes von digitalen Geräten in der Kita bestehen viele Vorbehalte, die jedoch häufig auch auf Unkenntnis hinsichtlich der Ziele und praktischen Umsetzung des Bildungsbereichs zurückzuführen sind. Ausgehend davon formulieren wir ebenfalls drei forschungsleitende Annahmen, die sich auf die Elternperspektive beziehen und im Folgenden analysiert werden:

- Annahme 2a: Die Mediennutzung (auch digitaler Medien) von Kindern im Kita-Alter hat während des ersten Lockdowns zugenommen.

- Annahme 2b: Dies hat bei den Eltern zu einer stärkeren Sensibilisierung für die Relevanz einer gezielten frühen Medienerziehung geführt.

- Annahme 2c: Die (ggf. positiven) Erfahrungen mit einer stärker digital ausgerichteten Kita haben zu Haltungsveränderungen hinsichtlich des Einsatzes digitaler Medien seitens der Kita zu 1) Kommunikationszwecken und 2) (medien)pädagogischen Zwecken geführt.

Die hier formulierten Annahmen beziehen sich sowohl auf die Perspektive der Erzieherinnen und Erzieher als auch auf die der Eltern als zentrale Akteurinnen und Akteure im Diskurs über die frühkindliche Medienerziehung. Die Coronapandemie, so lassen sich die hier formulierten und zu überprüfenden Annahmen zusammenfassen, könnte sich insofern als Gamechanger erweisen, als dass sie dem Diskurs über die frühkindliche Medienerziehung entscheidende Richtungsimpulse verleiht. 


\section{Methodisches Vorgehen}

\subsection{Aufbau der Studie}

Zwischen März und Juli 2020 wurde über das Umfragetool SoSci Survey eine nicht-repräsentative vollstandardisierte Online-Befragung mit Eltern $(n=154)$ und pädagogischen Fachkräften bzw. Leitungen ${ }^{3}(n=137)$ in zehn Kitas durchgeführt. Die Gruppen der Eltern wurden zur Rolle von digitalen Medien in ihrem familiären Alltag sowie ihren Haltungen zu Medienerziehung in der Kita befragt. Die Fachkräfte wurden zu ihren Fähigkeiten und ihrem Nutzungsverhalten von digitalen Medien (im privaten wie beruflichen Kontext) sowie ebenfalls zu ihren Haltungen befragt.

In einer zweiten Erhebungsphase von Juli bis August 2020 wurden die Leitungen bzw. Multiplikatorinnen und Multiplikatoren für Medienpädagogik der zehn Kitas mittels einer weiteren, teilstandardisierten Online-Befragung über das Tool Microsoft Forms zu ihren Tätigkeiten während des Lockdowns befragt, mit besonderem Fokus auf Formate des digitalen und nicht-digitalen Kontakts zu Eltern und Kindern. Die Ergebnisse wurden durch drei leitfadengestützte Einzelinterviews und eine Gruppendiskussion vertieft, die mit zwei Leitungen und drei Fachkräften geführt wurden. Hierfür wurden Einrichtungen kontaktiert, bei denen sich in der vorangegangenen Befragung Anknüpfungspunkte für tiefergehende Erläuterungen herauskristallisiert haben.

Im dritten Teil der Studie wurden im November und Dezember 2020 vier Gruppendiskussionen (Fokusgruppen) mit insgesamt neun Eltern aus vier Kitas (coronabedingt) mittels des Online-Videotools Zoom durchgeführt. Darin sollte eruiert werden, ob und inwiefern Eltern nach dem Lockdown eher den Bedarf nach gezielter Medienerziehung erkennen und sie digitale Angebote der Kita anders bewerten. Der Leitfaden umfasste u. a. Fragen zur Rolle von Medien im familiären Alltag (auch zur Zeit der coronabedingten Kontaktbeschränkungen und Kita-Schliessungen), Haltungen zu Medienerziehung in der Kita und zur Kita als Ansprechpartnerin für medienerzieherische Fragen (unter Einbeziehung möglicher mit dem Lockdown in Zusammenhang stehenden Haltungsänderungen) sowie Haltungen und Erfahrungen zu digitalen Kommunikationswegen zwischen der Kita und den Eltern bzw. Familien.

Die (deskriptive) Analyse der quantitativen Daten erfolgte mittels der Software STATA. Es wurde sich hier auf Häufigkeitsverteilungen sowie die Berechnung von Mittelwerte und Standardabweichungen beschränkt. Die freien Antwortfelder der Befragung wurden mittels induktiver Kategorienbildung nach Kuckartz (2018) ausgewertet.

3 Aus Anonymitätsgründen wurde nicht zwischen Leitungen und pädagogischen Fachkräften unterschieden, im Folgenden werden daher alle Beteiligten als „Fachkräfte” bezeichnet. 
Die Interviews und Gruppendiskussionen wurden orientiert an den inhaltlichsemantischen Transkriptionsregeln von Dresing und Pehl (2018) transkribiert und mittels der Datenanalysesoftware NVivo erfasst. Die qualitative Datenanalyse wurde an der strukturierenden qualitativen Inhaltsanalyse nach Kuckartz (2018, 97 ff.) orientiert. Dabei wurden sowohl induktiv als auch deduktiv Kategorien gebildet, mittels derer die Daten kodiert und ausgewertet wurden.

Fachpersonen \& Leitungen

$$
\begin{aligned}
& \text { vollstandardisierte Online-Befragung von Fach- } \\
& \text { personen und Leitungen aus zehn Kindertages- } \\
& \text { stätten }(n=137)
\end{aligned}
$$

Eltern

vollstandardisierte Online-Befragung von Eltern aus zehn Kindertagesstätten $(n=154)$

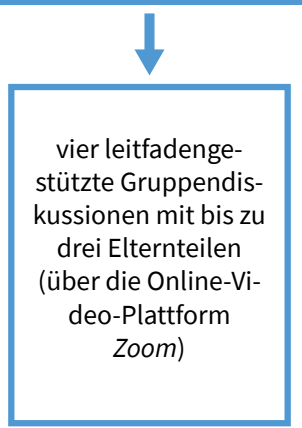

Abb. 1.: Darstellung des mehrstufigen methodischen Vorgehens (eigene Darstellung).

\subsection{Stichprobe}

Die hier vorgestellten Daten wurden im Rahmen des Forschungs- und Praxisprojekts «Medienerziehung im Dialog von Kita und Familie» in zehn Kitas aus drei Bundesländern erhoben, die sich - in sehr unterschiedlicher Intensität - bereits auf theoretischer Ebene mit dem Thema Medienerziehung auseinandergesetzt haben bzw. teilweise auch in der Praxis. ${ }^{4}$ Bei der Auswahl der zehn Einrichtungen wurden die Heterogenitätsmerkmale Lage (ländlich/suburban/urban), Grösse der Einrichtung, Trägerstruktur, Grad der Verankerung von Medienpädagogik im pädagogischen Alltag und im Kita-Konzept, Zusammensetzung des Teams und der Elternschaft berücksichtigt.

An der vollstandardisierten Online-Befragung haben in den beschriebenen zehn Kitas 154 Eltern und 137 pädagogischen Fachkräften bzw. Leitungen teilgenommen.

Der Link zur Befragung wurde über die Leitungen der zehn Kitas an alle Teammitglieder verteilt; die Eltern wurden auf unterschiedlichen Wegen auf die Befragung hingewiesen, entsprechend der üblichen Kommunikationswege in den jeweiligen

4 Für die Teilnahme an dem Forschungs- und Praxisprojekt war ein Bewerbungsverfahren notwendig; die Kitas werden über einen Zeitraum von zwei Jahren begleitet (von Herbst 2019 bis Herbst 2021) und mit einer Summe in Höhe von 5.000 Euro gefördert. 
Einrichtungen (per E-Mail, über Messengergruppen der Elternvertretenden, über Aushänge bzw. durch gezielte Ansprache durch die Fachkräfte). Die Rücklaufquote lag unter den Fachkräften im Schnitt bei 57 \% (die Werte variieren je nach Einrichtung stark und liegen zwischen $36 \%$ und $94 \%$ ). In den zehn Einrichtungen arbeiten insgesamt 239 pädagogische Fachkräfte und Leitungen, die Teamgrösse der jeweiligen Einrichtungen variiert zwischen 14 und 44 Teammitgliedern. Die Rücklaufquote in der Gruppe der Eltern kann weniger genau angegeben werden. Insgesamt werden in den zehn Kitas 1.377 Kinder betreut, worunter jedoch ein unbekannter Anteil an Geschwisterkindern und Kinder von Alleinerziehenden fällt, sodass die genaue Anzahl potenziell erreichbarer Eltern nicht eindeutig zu benennen ist. Deutlich ist jedoch, dass die Rücklaufquote in der Gruppe der Eltern deutlich geringer ausfällt, als in der Gruppe der Fachkräfte.

Das Sample ist mehrheitlich weiblich (86\% der Fachkräfte und $80 \%$ der Eltern). Die Alterszusammensetzung ist unter den Fachkräften erwartungsgemäss breiter gefächert als unter den Eltern. Letztere sind zu rund $86 \%$ zwischen 25 und 45 Jahren alt; unter den Fachkräften sind es nur $53 \%$ (siehe Abb. 2) ) $^{5}$. Die befragten Fachkräfte sind vorrangig im Elementarbereich tätig (65 \%); $40 \%$ sind im Krippen- bzw. Nestbereich tätig, $29 \%$ im Vorschul- und acht Prozent im Hortbereich (Mehrfachantworten waren möglich) ${ }^{6}$. Eine wichtige Besonderheit der Samples ist, dass $21 \%$ ( $n=29$ aus neun von 10 Kitas) der befragten Fachkräfte in ihrer Kita eine Multiplikatorinnen und Multiplikatoren-Rolle für das Thema Medienpädagogik innehaben. Diese Zuordnung wurde von den Fachkräften selbst anhand einer entsprechenden vorgenommen.

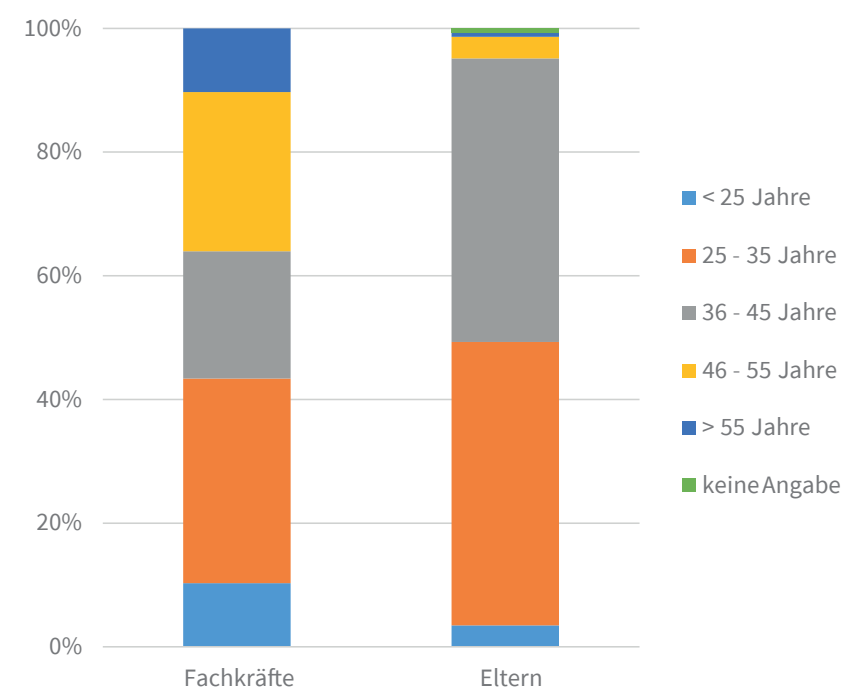

Abb. 2.: Altersstruktur der befragten Fachkräfte $(n=137)$ und Eltern $(n=154)$; Angaben in \%.

5 Aus Anonymitätsgründen wurde nicht das genaue Alter erhoben, sondern lediglich die Einordnung in eine von fünf Altersklassen erfragt.

6 Der Gesamtdatensatz wurde um einen Datensatz bereinigt, da in diesem der Fragebogen lediglich zu $<10 \%$ ausgefüllt wurde. Alle übrigen Datensätze $(n=137)$ liegen vollständig vor. 
Die befragten Eltern haben im Schnitt 1,7 Kinder $(S D=0,84)$, die im Mittel 3,8 Jahre alt sind $(S D=1,47)$. Sie sind laut Selbsteinschätzung finanziell überdurchschnittlich gut abgesichert $(M=6,95 ; S D=1,69$ auf einer Skala von $1=$ «nicht gut» bis $10=$ «sehr gute finanzielle Situation»). $16 \%$ der Eltern sind alleinerziehend. Nahezu alle Eltern sprechen Deutsch mit ihren Kindern, 30 \% sprechen eine oder mehrere zusätzliche Sprachen zu Hause. Die am häufigsten gesprochene Sprache ausser Deutsch ist Englisch $(n=30)$. Mit $42 \%$ ist der Hochschulabschluss (oder die Promotion) der häufigste höchste Bildungsabschluss der Befragten (siehe Abb. 3).

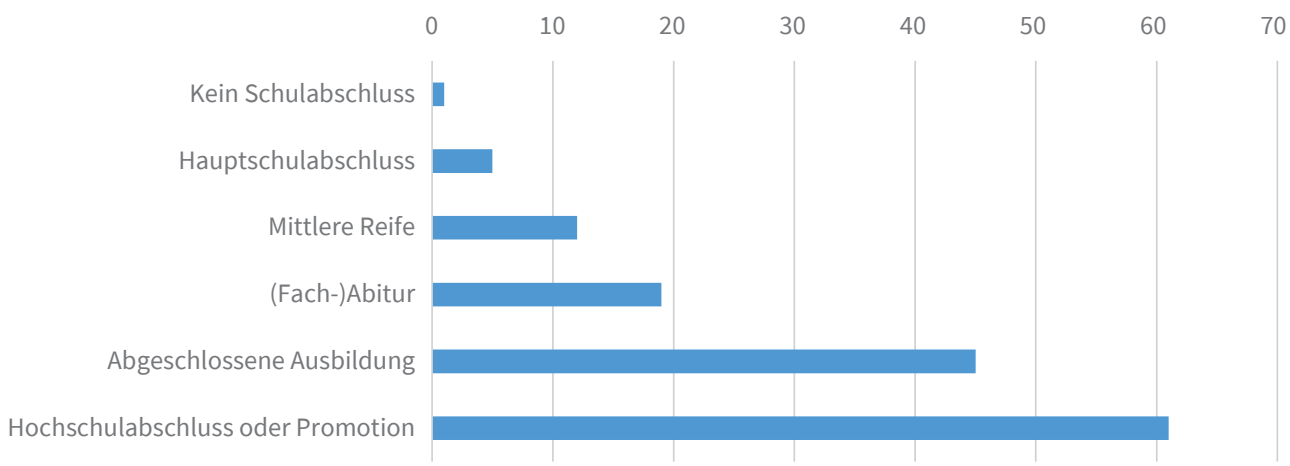

Abb. 3.: Höchster Bildungsabschluss der befragten Eltern ( $n=143)$.

An den teilstandardisierten Online-Befragungen (via Microsoft Forms) sowie die ergänzenden bzw. vertiefenden Telefoninterviews haben Leitungen bzw. medienpädagogische Multiplikatorinnen und Multiplikatoren teilgenommen. Erstere wurde als Link an alle zehn Einrichtungen geschickt, welche selbstständig entschieden haben, von wem die Fragen beantwortet werden (eine Kita hat die Befragung von zwei unabhängigen Personen beantworten lassen). An den anschliessenden Telefoninterviews (drei leitfadengestützte Einzelinterviews und eine Gruppendiskussion) haben zwei Leitungen und drei pädagogische Fachkräfte (medienpädagogische Multiplikatorinnen und Multiplikatoren) teilgenommen. Da hier die Auskunft zu Prozessen und Entwicklungen innerhalb der jeweiligen Kitas im Vordergrund stand - und nicht die persönlichen Haltungen der Befragten bzw. Interviewten -, wurden an dieser Stelle keine soziodemografischen Daten erhoben.

Die Teilnahme an den Gruppendiskussionen erfolgte freiwillig, die entsprechende Einladung wurde über die Kita-Leitungen bzw. Elternvertretenden an die Eltern der Einrichtungen kommuniziert. An den Gruppendiskussionen haben pro Termin mit einer Ausnahme zwei oder drei Eltern teilgenommen. Das Geschlechterverhältnis war relativ ausgeglichen: insgesamt wurden fünf Mütter und vier Väter befragt. Die teilnehmenden Eltern haben ein Durchschnittsalter von 38,6 Jahren und einen mittleren bis hohen sozioökonomischen Status und lassen sich als digital kompetent bis 
affin beschreiben. Sie verfügen mindestens über eine abgeschlossene Ausbildung, grösstenteils jedoch über einen Hochschulabschluss. Sie haben im Durchschnitt 1,3 Kinder $(S D=0,7)$ im Alter von 19 Monaten bis acht Jahren. ${ }^{7}$

\section{Ergebnisse}

Die Ergebnisse werden im Folgenden zur leichteren Nachvollziehbarkeit thematisch zusammengefasst. Dabei wird zunächst auf die für Fachkräfte bzw. Leitungen formulierten Annahmen Bezug genommen, anschliessend auf die für die Gruppe der Eltern. Die Ergebnisse aus der vollstandardisierten Online-Befragung von Eltern und Fachkräften dienen hier als Grundlage, auf Basis derer die Ergebnisse der qualitativen Teilstudien (teilstandardisierte Befragung und leitfadengestützte Interviews mit Leitungen und Fachkräften bzw. Gruppendiskussionen und Einzelinterview mit Eltern) vor allem in Bezug auf die coronabedingten Veränderungen eingeordnet werden. Die Interviews dienen nicht nur der Illustration der Ergebnisse der quantitativen Befragung, sondern liefern darüber hinaus tiefergehende Erkenntnisse und Hinweise auf weitere, aus den quantitativen Daten nicht hervorgehende Relevanzstrukturen.

\subsection{Perspektive der pädagogischen Fachkräfte und Leitungen}

\section{Zu Annahme $1 a$}

Unserer ersten Annahme (1a) zufolge haben die Kindertagesstätten während der Pandemie diverse Prozesse, die im normalen Alltag analog ablaufen, teilweise oder vollständig über digitale Wege realisiert. Die Ergebnisse legen nahe, dass die Kitas im ersten Lockdown vor allem die Kommunikation mit Eltern stärker digital ausgerichtet haben. In einigen Einrichtungen wurden auch Rituale aus dem pädagogischen Alltag in digitale Formate übertragen.

Die quantitative Befragung der Fachkräfte und Leitungen zu Beginn der Pandemie deutete zunächst darauf hin, dass digitale Medien in ihrem Arbeitsalltag keine unwesentliche Rolle spielen, jedoch insbesondere die Kommunikation mit Eltern noch sehr analog stattfindet. Nur ein Viertel ( $26 \%)$ der Befragten geben an, hierfür digitale Medien einzusetzen, etwa für die Kommunikation per E-Mail (siehe Abb. 4).

7 Die soziodemographischen Daten wurden im Anschluss an die Gruppendiskussionen mittels einer kurzen vollstandardisierten Onlinebefragung über das Tool Microsoft Forms erhoben. 


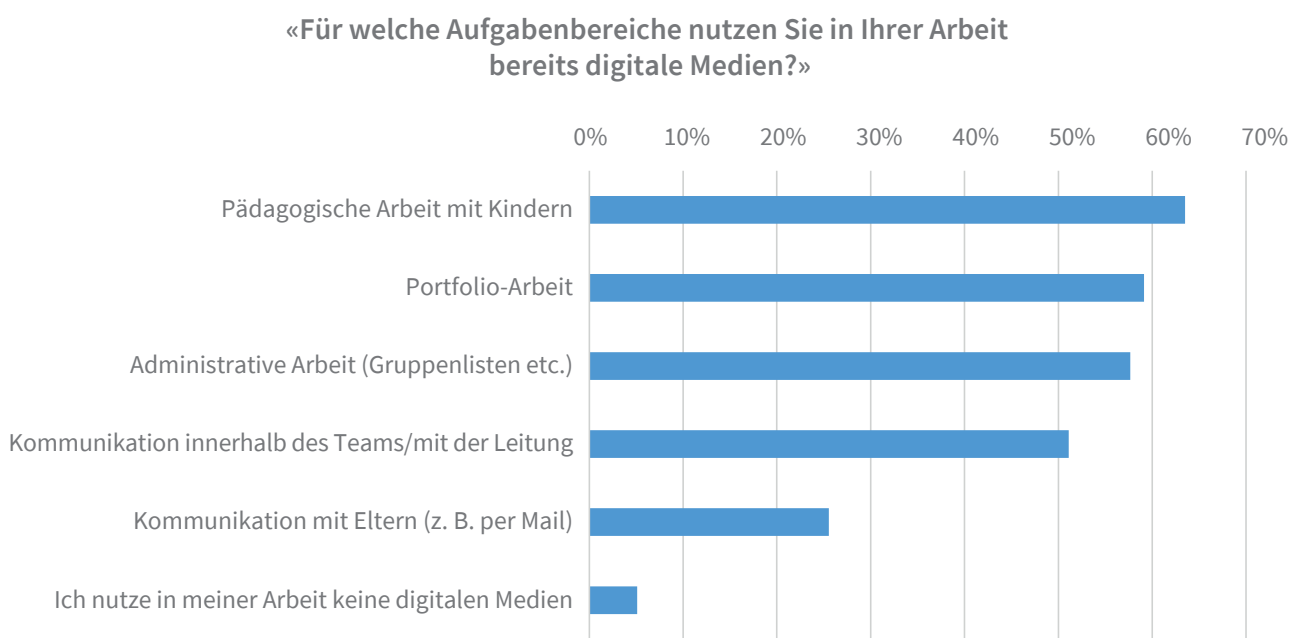

Abb. 4.: Zwecke der Nutzung digitaler Medien seitens der befragten Fachkräfte im Arbeitskontext (Angaben in \%; Mehrfachnennung möglich).

In der im Juli und August 2020 durchgeführten teilstandardisierten Online-Befragung wurden von den Kitas hingegen diverse neue Formate und Wege genannt, mit denen sie seit dem Lockdown mit den Familien und Eltern in Kontakt stehen. Sechs der zehn Kitas geben an, digitale Medien während des ersten Lockdowns genutzt zu haben, um den Kontakt zu den Familien nicht abreissen zu lassen.

Besonders auffällig ist das grosse Repertoire an Kommunikationswegen, welche von den Kitas erstmalig ausprobiert wurden. So wurden E-Mail-Verteiler oder Newsletter erstellt, um den Eltern Informationen zukommen zu lassen. Auch klassische Formate wie Elternratssitzungen, Elterngespräche und Elternabende, aber auch speziellere Formate wie die Elternberatung wurden per Videokonferenz ins Digitale übertragen. ${ }^{8}$ Teils wurden pädagogische Anregungen wie Mal- und Bastelanleitungen in Clouds oder Padlets (eine Art digitale Pinnwand) bereitgestellt. Mit einigen Vorschulkindern wurden Videocalls durchgeführt; auch digitale Morgenkreise, von den Fachkräften gedrehte Videos (mit Vorlesegeschichten, kleinen Experimenten oder Geburtstagsgrüssen) sowie E-Mails an die Kinder gehörten zu den genannten Formaten. Eine (medienpädagogisch bereits recht aktive) Kita hat die festen Termine ihres pädagogischen Alltags ins Digitale verlagert und fast täglich Videos mit den Familien geteilt: von Sportangeboten über Morgenandachten bis hin zu Mitmachliedern, aber auch Informationen an Eltern (etwa zur Mediennutzung zu Hause und empfehlenswerten Kinder-Apps). Eine Fachkraft dieser Einrichtung beschreibt den Hintergrund dieses Vorgehens folgendermassen:

«Wir sind ja auch ein Familienzentrum, und da war die Situation wirklich ganz absurd, wenn man überhaupt keinen Kontakt mehr halten kann, persönlich.»

8 In zwei Kitas gab es seitens des Trägers die Möglichkeit für Eltern und Familien, sich zwecks Aufrechterhaltung des Kontakts zur Kita ein Tablet auszuleihen. 
Es standen jedoch nicht alle Kitas digital mit den Eltern in Kontakt. Eine Kita gibt an, zwar Ideen für diesen Austausch gehabt zu haben, aber noch nicht das Know-how zu besitzen, diese umzusetzen. Zudem erschwere die veraltete technische Ausstattung digitale Möglichkeiten der Kontaktaufnahme. Eine andere (medienpädagogisch recht aktive) Kita schätzte den Unterstützungsbedarf ihrer Elternschaft (die über einen tendenziell hohen sozioökonomischen Status verfügen) während des Lockdowns als niedrig ein. Zudem fürchtete sie eine starke Erwartungshaltung seitens der Eltern, die sich mit den vorhandenen Ressourcen kaum bewältigen lasse und die einer digitalen Rundum-Betreuung der Kinder gleichkommen würde. Daher hielten sie sich - obwohl die Kita über eine überdurchschnittlich gute technische Ausstattung verfügt - im Kontakt mit den Eltern (und Familien) eher zurück.

Eine weitere befragte Kita lehnt die digitalen Kommunikationswege - insbesondere mit Kindern - explizit ab. Eine Fachkraft berichtet im Rahmen eines Interviews, von einer Elternvertreterin nach digitalen Morgenkreisen gefragt worden zu sein, die sie jedoch nicht durchführen wolle:

«Ein Morgenkreis ist ein Morgenkreis, den macht man hier.»

Die Leitung der Kita fügt hinzu:

«Da bin ich auch so'n bisschen zwiespältig. [...] Das wurde in den Medien gebracht, dieses digitale Bilderbuch-Vorlesen. [...] Aber ick sach, nee Leute irgendwo, weiß ich nicht. Was hat das Kind davon, wenn es jetzt vor'm Bildschirm sitzt und kann das Buch nicht anfassen und kann nicht sagen «Ich hab's nicht verstanden» oder «Ich will die Zeile nochmal hören».

Fast alle Kita-Leitungen geben an, eine hohe Belastung durch die sich häufig ändernden coronabezogenen Vorgaben zu erfahren und kaum über Kapazitäten für zusätzliche Aufgaben zu verfügen.

\section{Zu Annahme $1 b$}

Der zweiten Annahme (1b) zufolge haben die pädagogischen Fachkräfte durch die vermehrte Nutzung digitaler Medien ihre Kompetenzen im Umgang mit digitalen Medien ausgebaut. Die Ergebnisse zeigen, dass viele Fachkräfte ihr Mediennutzungsrepertoire in den lockdownbedingten Schliesszeiten erweitert haben. Digitale Medien wurden für vielseitige Zwecke genutzt: zu organisatorischen, pädagogischen, Bildungs- und Kommunikationszwecken sowie auf praktischer wie theoretischer Ebene.

Die quantitative Befragung von pädagogischen Fachkräften und Leitungen in den zehn Kitas $(n=137)$ hat ergeben, dass sich diese gegenüber der ebenfalls befragten Gruppe an Eltern $(n=154)$ kaum hinsichtlich ihrer Mediennutzung unterscheiden. 
Beide Gruppen schätzen die Rolle, die digitale Medien in ihrem Leben spielen, als gleichwertig hoch ein (Fachkräfte: $M=3,97, S D=0,89$, Eltern: $M=3,90, S D=0,98$ auf einer Skala von $1=$ «gar keine Rolle» bis $5=$ «eine große Rolle»). Die Fachkräfte geben für die regelmässige private Nutzung digitaler Medien in allen abgefragten Bereichen (Unterhaltung, Kommunikation, Social Media, Information, Online-Shopping, Organisation) im Mittel sogar leicht höhere Werte als die (im Schnitt jüngere) Gruppe der Eltern an, insbesondere im Bereich Unterhaltung.

Trotz der scheinbar hohen Mediennutzung persistieren unter den Fachkräften teilweise Unsicherheiten im Umgang mit digitalen Geräten. Ein Viertel gibt an, nicht ohne Hilfe ein E-Mail-Konto anlegen zu können, knapp 30 \% haben Probleme beim Übertragen von Dateien zwischen zwei Geräten. Insbesondere der kreativ-gestalterische Bereich stellt hier offenbar eine Herausforderung dar: $37 \%$ der Fachkräfte geben an, sich Foto- und Bildbearbeitung nicht oder nur mit Hilfe zuzutrauen, bei der Videobearbeitung gilt dies für $73 \%$.

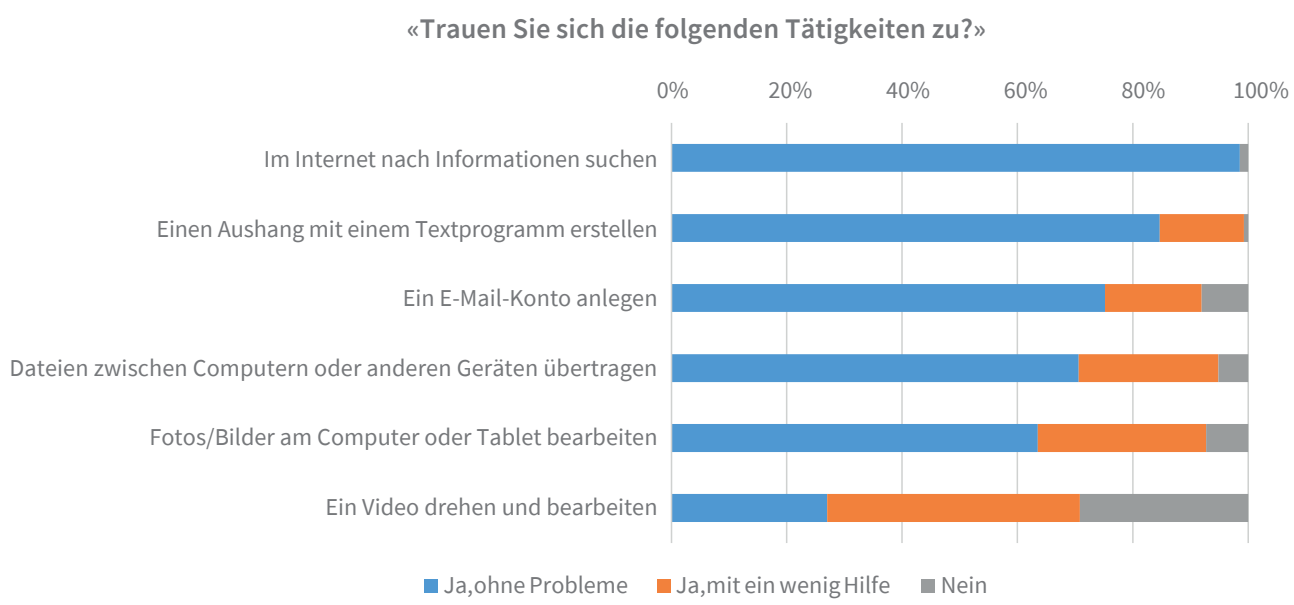

Abb. 5.: Selbsteinschätzung zu Tätigkeiten mit digitalen Medien seitens der befragten Fachkräfte; Angaben in Prozent ( $n=137)$.

In der Coronapandemie wurden einige Fachkräfte mit neuen Herausforderungen im Umgang mit digitalen Medien konfrontiert. Zu ihren Aktivitäten im ersten Lockdown befragt, geben einige der Fachkräfte und Leitungen an, dass digitale Medien über die Kommunikation mit Eltern und Kindern (teilweise auch über selbstgedrehte Videos o. ä., siehe oben) hinaus auch zur Recherche pädagogischer Materialien oder zu Online-Fortbildungen genutzt wurden. In einem vertiefenden Interview beschreibt eine Kita-Leitung ihre erste Erfahrung mit einer Videokonferenz zunächst als Herausforderung:

«Na weil wir's ja nicht brauchen, ist ja klar. Wir haben das ja noch nie gemacht.» 
Die Erfahrungen waren jedoch positiv, ihre Kollegin resümiert:

«Dann haben wir es doch noch geschafft irgendwie. [...] Aber war ja witzig, hat ja dann gut geklappt.»

Eine andere Fachkraft berichtet jedoch auch von Online-Fortbildungsformaten, die aufgrund mangelnder Expertise aufseiten der Anbieter weniger gewinnbringend waren. Eine Leitung sagt im Interview, dass sie und ihr Team viel dazugelernt hätten und nun souveräner im Umgang mit den Geräten seien.

Darüber hinaus geben neun der zehn Kitas an, sich während des Lockdowns mit dem Thema Medienpädagogik auf theoretischer Ebene auseinandergesetzt zu haben - etwa in Bezug auf die Verankerung im Kita-Konzept und hinsichtlich der Frage nach sinnvollen technischen Anschaffungen, oder für die Planung themenbezogener Fachtage und/oder Teamschulungen als auch Recherche nach guten Materialien.

Einzelne Kitas haben in Zeiten der Notbetreuung auch ihre praktische medienpädagogische Arbeit ausgebaut. Hier ist die Aussage einer interviewten Fachkraft interessant, dass die kleinen Gruppengrössen und der infolgedessen höhere Betreuungsschlüssel hilfreich waren, um die (im Rahmen des Projekts) neu angeschafften digitalen Geräte zunächst mit wenigen Kindern zu testen.

\section{Zu Annahme 1c}

Annahme 1c zufolge hat die grössere Sicherheit im Umgang mit digitalen Geräten zu einem Abbau von Vorbehalten hinsichtlich deren Einsatzes in ihrer Arbeit geführt. Die Ergebnisse der Untersuchung deuten darauf hin, dass die neuen Erfahrungen mit digitalen Medien von den Fachkräften zwar positiv aufgenommen werden, jedoch nicht zwangsläufig zu einer höheren Sensibilisierung für die Notwendigkeit von Medienerziehung führen.

Die vollstandardisierte Onlinebefragung zu Beginn des ersten Lockdowns zeigt, dass bei der Mehrheit der Fachkräfte kein klares Verständnis von Medienerziehung vorhanden ist - wenngleich sich ihre Kita bereits mit der Thematik auseinandergesetzt hat. Zwar gibt lediglich ein kleiner Teil der Fachkräfte an, dieser skeptisch oder ablehnend gegenüberzustehen (11\%) und gut die Hälfte findet diese wichtig. Nach einem Beispiel für (gute) Medienerziehung in der Kita befragt gibt nur gut ein Drittel der Fachkräfte an, ein solches Beispiel geben zu können (gegenüber ca. einem Viertel der Eltern, s.u.). Jeweils ca. ein Drittel gibt an, dahingehend unsicher zu sein bzw. kein Beispiel nennen zu können. Die genannten Beispiele $(n=51)$ lassen ein relativ breites Antwortspektrum erkennen, welches differenzierter ist als das der Eltern (s.u.). Im Fokus steht häufig die nicht-konsumorientierte Nutzung der Geräte, die Begleitung der Kinder durch die Fachkräfte, Aufgreifen des (mediatisierten) Alltags der Kinder sowie das Aufzeigen der Vielfalt der Medienlandschaft. Zusammenfassend 
lässt sich dazu festhalten, dass obwohl einzelne Fachkräfte bereits ein recht gutes Verständnis von Medienerziehung erkennen lassen, das Wissen noch nicht in der Breite der Teams verankert ist.

Ob die Umbrüche im Kita-Alltag, welche die Coronapandemie nach sich zog zu langfristigen Veränderungen und unter Fachkräften zu einer stärkeren Sensibilisierung für die Notwendigkeit von Medienpädagogik führt, wird von den Kitas unterschiedlich eingeschätzt. Insgesamt wurden viele Änderungen eher ad hoc durchgeführt und waren weniger auf eine langfristige Verankerung ausgelegt. Eine Fachkraft reflektiert die Lage folgendermassen:

"Es wird sich auch noch zeigen, was von der Corona-Krise übriggeblieben ist, von den ganzen Sachen, die man jetzt angefangen hat mit den Medien, ob es sich nicht lohnt, da noch Sachen weiter auszubauen und weiter aufrechtzuerhalten.»

Hinsichtlich der Haltungen der Fachkräfte sagt die gleiche Fachkraft: «Es ist ganz viel im Umbruch und durch Corona wird sich bei uns glaube ich auch die medienpädagogische Einstellung ändern.»

Ein anderer Erzieher, der in seiner Kita Multiplikator für das Thema Medien ist, vertritt ebenfalls die Ansicht, dass der überwiegende Teil des Kollegiums in der Zeit offener geworden sei, gibt jedoch auch zu bedenken:

«der ein oder andere wird schon noch abgeschreckt sein, weil ja viel Technik da ist».

Positiv bewertet werden Änderungen insbesondere dort, wo sie merkliche Vorteile bieten. Digitale Elternratssitzungen schaffen etwa mehr zeitliche und räumliche Flexibilität. Eine Fachkraft führt im Interview aus:

«Wenn man die [Eltern, Anm. d. Verf.] hier im Haus hat, dann muss man immer gucken, wann können alle hier sein, dann kommt einer 10 Minuten später, das ist dann manchmal schwierig, dann findet man keinen Termin. Aber da können sie sich, wo auch immer sie sind, einschalten und das ist gar nicht verkehrt. Da ist man flexibler einfach.»

Ergänzend fügt sie an, per E-Mail nun auch Eltern zu erreichen, die sonst sehr passiv und wenig aufgeschlossen gewesen seien. Auch digitale Fortbildungsangebote wurden von einigen Fachkräften als positiv eingeschätzt - sofern sie ohne technische Probleme abliefen.

Kitas, die im Umgang mit digitalen Medien eher Hemmungen verspüren, bewerten jedoch auch die Sinnhaftigkeit digitaler Kommunikation mit Eltern und Kindern als geringer. Sie heben die Nachteile besonders hervor - etwa wie oben bezüglich der 
Ortsgebundenheit des Morgenkreises («den macht man hier»), des pädagogischen Werts von Vorlesevideos («was hat das Kind davon») oder auch bezüglich digitaler Elternabende:

«Das würde die [Eltern, Anm. d. Verf.] sicher abschrecken».

Auch Einrichtungen, die während des ersten Corona-Lockdowns weniger digitale Medien für den Kontakt zu den Familien nutzten, kennen die in den Medien behandelten Beispiele von Kitas, die während der Schliesszeiten stärker digital ausgerichtet waren (über Morgenkreise, Videos, Online-Konferenzen o. ä.) und wurden teilweise von ihrer Elternschaft darauf angesprochen. Diese Forderungen seitens der Eltern können zukünftig möglicherweise zu schrittweisen Haltungsänderungen führen, was bislang jedoch noch nicht erkennbar ist.

In der teilstandardisierten Befragung äussern nur zwei der zehn Kitas den Eindruck, dass die Eltern durch die veränderten Lebensbedingungen im Kontext der Covid-19-Pandemie ein höheres Interesse am Thema Medienpädagogik haben. Einige Fachkräfte drücken die Vermutung aus, dass sich die Medien-, insbesondere Fernsehnutzung der Kinder während des Lockdowns deutlich erhöht hat, diese Annahme ergab sich teilweise aus dem beobachteten Verhalten der Kinder (z. B. im Freispiel).

\subsection{Perspektive der Eltern}

\section{Zu Annahme $2 a$}

Angesichts der veränderten Ausgangslage während des ersten Lockdowns wurde eine Zunahme der Mediennutzung von Kindern im Kita-Alter angenommen (Annahme 2a). Die Ergebnisse zeigen, dass digitale Medien während des Lockdowns verstärkt bei Kindern ab drei Jahren zur Alltagsbewältigung eingesetzt werden.

Die vollstandardisierte Online-Befragung zeigt, dass die Kita-Kinder der befragten Eltern in ihrem Alltag diverse digitale Medien nutzen, wenngleich sie nicht auf das volle Repertoire der Medienausstattung des Haushalts Zugriff haben. Die Kinder nutzen im Mittel 2,02 digitale Geräte $(S D=0,98)$, während im Haushalt durchschnittlich 3,55 Geräte $(S D=1,31)$ vorhanden sind.

Nach der Bedeutung digitaler Medien in ihrem Familienalltag befragt, geben insbesondere Eltern mit kleinen Kindern häufig an, Medien vermeiden zu wollen. Bei Eltern von 3-jährigen Kindern sagen dies jedoch nur noch die Hälfte der Eltern - der Anteil nimmt mit steigendem Alter der Kinder sukzessive ab und liegt bei Eltern von 6-Jährigen nur noch bei ca. $12 \%$.

Der Einfluss des Kindesalters auf die Mediennutzung des Kindes zeigt sich auch in den Gruppendiskussionen deutlich: In den Familien mit Kindern im Alter von ein bis zwei Jahren ist die Mediennutzung der Kinder im ersten Lockdown unverändert 
geblieben, da diese ohnehin keine oder kaum Medien rezipieren. ${ }^{9}$ In allen befragten Familien mit Kindern ab drei Jahren hat die Mediennutzung der Kinder - insbesondere die Nutzung von (Smart-)Fernsehern und Tablets - hingegen zugenommen.

Der Zusammenhang zwischen dem Alter des Kindes und der Vermeidung von Mediennutzung wurde von einigen Elternteilen reflektiert:

«Ich find das hängt auch mit dem Alter [...] [des Kindes, Anm. d. Verf.] zusammen, wie man selbst erlebt, wie das Kind immer mehr wissen möchte und erfahren möchte und auch einfach dazu bereit ist, immer mehr aufzunehmen [...].» (Mutter eines 3-jährigen Kindes, 28 Jahre)

«Vor einem Jahr hätte ich mir nicht vorstellen können, dass sie schon so viel macht und so viel aufnimmt [...]. Man muss ja auch gefördert werden und [...] die Entwicklung geht in diese Richtung und wir können die Kinder nicht davon [von digitalen Medien, Anm. d. Verf.] fernhalten.» (Vater eines 1,5-Jahre alten Kindes, 50 Jahre)

\section{Zu Annahme $2 b$}

Annahme $2 b$ besagt, dass die während der Pandemie erhöhte Mediennutzung der Kinder zu einer stärkeren Sensibilisierung für die Relevanz einer gezielten frühen Mediennutzung führt. Dies konnte für die Mehrzahl der Fälle nicht bestätigt werden. So verbinden Eltern die angestiegene Mediennutzung nur selten mit einem höheren Bedarf an einer gezielten Medienerziehung.

Die Ergebnisse der vollstandardisierten Online-Befragung zeigen, dass die meisten Eltern keine genaue Vorstellung von dem Sinn und Zweck einer gezielten Medienerziehung haben. Fast drei Viertel der Eltern können kein Beispiel für gute Medienerziehung in der Kita geben (im Vergleich zu knapp zwei Drittel der Fachkräfte, s. o.). Die genannten Beispiele $(n=38)$ zeigen, dass die Befragten eher basierend auf ihren Alltagserfahrungen (die sich im Wesentlichen auf Regulierung und Einschränkung der kindlichen Mediennutzung beziehen) auf das Thema blicken. So geben sie zwar mehrheitlich an, Medienerziehung in der Kita für sinnvoll zu erachten (s. o.), erwarten von dieser jedoch vor allem den (zeitlich) eingeschränkten Einsatz von Medien z. B. zwecks Wissensvermittlung (in Form von Lernspielen o. ä.).

Die vermehrte Mediennutzung der Kinder ab drei Jahren hat während des ersten Lockdowns unter den Teilnehmenden der Gruppendiskussion nicht erkennbar zu einer höheren Sensibilisierung für das Thema Medienerziehung geführt. Die stärkere Nutzung wurde von den Eltern weniger in Bezug auf die Bedarfe der Kinder, denn vor dem Hintergrund der eigenen Bedarfe reflektiert. Auch hier steht also eher der (von den Eltern weniger streng verfolgte) Regulierungsaspekt im Vordergrund, anstelle einer Befähigung des Kindes.

9 Eine Ausnahme stellt teilweise die Videotelefonie mit Verwandten, Freundinnen oder Freunden dar, die jedoch als unproblematische Mediennutzung bewertet wurde. 
In allen Fällen der angestiegenen Mediennutzung wurde als Begründung angeführt, dass diese die Bewältigung des Alltags, in den meisten Fällen Homeoffice oder die Erledigung von Aufgaben im Haushalt, erleichtert oder überhaupt erst ermöglicht habe.

«Die Mediennutzung hat zugenommen, definitiv. Weil wir beide im Homeoffice arbeiten können. Das ist ein großer Vorteil, den ich da sehe. Gerade auch, wenn die Kita zu war.» (Vater eines Kindes, 45 Jahre)

Auf der anderen Seite zeigte sich, dass der Medieneinsatz zwar als pragmatische (temporäre) Lösung angesehen wurde, aber dennoch mit einer (selbst-)kritischen Haltung und Schuldgefühlen einherging.

«Bei uns war es definitiv so [dass die Mediennutzung zugenommen hat, Anm. d. Verf.] und ich muss sagen, ich fand das aber nicht gut. [...] Mein Mann und ich haben beide im Homeoffice gearbeitet [...] und hatten halt drei Kinder hier zu Hause. [...] Der Größte musste Schulaufgaben machen, aber die anderen beiden, die musste man zwischendurch auch einfach mal kurz stillstellen, sag ich jetzt einfach mal.» (Dreifache Mutter, 36 Jahre)

«[1]mmer konsequent sein fällt natürlich schwer. Auch wenn man bestimmte Sachen einfach fertigkriegen will und das [...] durch den Lockdown [...] nochmal verschärft ist, dass man dann sagt "Okay und jetzt mal kurz eine halbe Stunde vor den Computer» und ja, das ist natürlich nicht toll, das weiß man irgendwie selber [...]. [D]a könnte man vielleicht sich mehr Zeit nehmen und erklären ‘Du, Mama muss das jetzt mal kurz machen und mach doch in der Zeit das und das', das ist dann einfach irgendwie gerade nicht drin.» (Mutter eines Kindes, 42 Jahre)

«Man merkt, wenn sie [die Tochter, Anm. d. Verf.] länger davorsitzt, egal, was sie da guckt, dass sie dann ab 17, $18 \mathrm{Uhr}$ manchmal unausstehlich wird [...] und dahingehend hin ist es schon eine Belastung für die Eltern auch beide, wenn man sich dann wieder vorwirft, warum hast du schon wieder so viele Termine gehabt heute.» (Vater eines Kindes, 45 Jahre)

\section{Zu Annahme 2c (1)}

Die letzte für die Elternseite formulierte Annahme (2c) wurde in zwei Unterpunkte unterteilt. Ersterer besagt, dass eine stärker digital ausgerichtete Kita zu einer Veränderung der Haltungen von Eltern gegenüber dem Einsatz digitaler Medien zu Kommunikationszwecken führt. Diese Annahme hat sich bestätigt: So besteht aufgrund der positiven Erfahrungen mit digitalen Kommunikationswegen zwischen Kita und 
Eltern seitens der Eltern der Wunsch nach einer verstärkten digitalen Kommunikation. Einige Eltern beziehen diese Forderung auch auf die Kommunikation mit den Kindern.

Wie oben bereits geschildert, sind viele Einrichtungen während des ersten coronabedingten Lockdowns auf digitale Kommunikationswege ausgewichen, um Kontakt mit den Familien und Kindern zu halten. In den Gruppendiskussionen haben sich nach diesen Erfahrungen acht der neun Eltern für eine verstärkte E-Mail-Kommunikation seitens der Kita ausgesprochen. Zwar wurde in mehreren Fällen betont, dass kindbezogene Informationen weiterhin im persönlichen Gespräch kommuniziert werden sollten, E-Mails wurden hingegen als schnellere und effizientere Alternative zu schriftlichen Aushängen gesehen.

"[D]as ist das Paradoxe an der Situation, dadurch, dass man sich eigentlich überhaupt nicht mehr sieht ist trotzdem gefühlt die Kommunikation besser geworden, weil viel mehr über E-Mail ausgetauscht wird [...] also ich fühle mich aktuell viel stärker abgeholt, wie vor dem Lockdown, da hatten wir teilweise noch nicht mal E-Mail-Verteiler.» (Zweifacher Vater, 45 Jahre)

"Also ich fände es auch gut, wenn es mehr digitalisiert wird, weil es gibt so gute und einfache Mittel mit einem Knopfdruck alle zu informieren, die betroffen sind, das geht sehr simpel, [...] und es würde auch der Kita viel Zeit und Rückfragen sparen glaube ich.» (Vater eines Kindes, 50 Jahre)

Während fast flächendeckend der Wunsch nach einer verstärkten digitalen Kommunikation geäussert wird, sind die Meinungen zur digitalen Kommunikation der Kita mit den Kindern (über Videos, Morgenkreise, Videocalls etc.) durchmischter. Einige Eltern äussern keinen entsprechenden Bedarf oder erachten diese für eher unwichtig bzw. nicht sinnvoll:

«Also für mich ist zum Beispiel nicht so wichtig, dass jetzt die Erzieherinnen für uns da ein Video aufnehmen. Es gab mal diese Aktion, dass alle Kinder einen Stein bemalen sollen und vor die Kita hinlegen, also auch sehr analog. Aber irgendwie war mir wichtig / man war gut aufgehoben, man war gut informiert, es wird auf die Bedürfnisse reagiert, einfach eine gute Kommunikation.» (Mutter eines Kindes, 42 Jahre)

Kontrastierend bewerten vier der neun befragten Eltern die kindbezogenen digitalen Angebote als äusserst positiv und äussern den Wunsch, dass diese - im Kontext von Corona - verstärkt angeboten werden. 
"[W]ährend der Coronazeit, da wurde mit den Schulanfängern so ein ZoomTreffen aufgebaut und ich muss sagen, meine Tochter war auch mega begeistert, da hat sie sich hingesetzt und meinte «ja Mama, heute habe ich auch einen Termin» und war ganz stolz [...], dann ist es aber auch bei dem einen Termin geblieben [...], das fand ich dann auch schade, dass man das dann nicht beibehält.» (Dreifache Mutter, 36 Jahre)

«Also ich würde es auf jeden Fall sehr positiv bewerten, sollte es nochmal zu so 'nem Lockdown wie im Frühjahr kommen, dass es mehr wäre als letztes Mal. Weil eigentlich kam von der Kita gar nichts, ausser einer Sprachnachricht über WhatsApp mit 'nem Kitalied, das war sehr süss und da haben die Kleinen sich auf jeden Fall sehr gefreut, mal was von ihren Erziehern zu hören. Aber das hat so gefehlt, die Kita und die anderen Kinder, also irgendein Austausch, sei es einmal die Woche ein kurzer Videocall [...], dass die Kinder merken, okay die sind noch da, weil das war echt schon 'ne harte Zeit.» (Mutter eines Kindes, 28 Jahre)

Teilweise haben die Eltern über die publizistischen Medien wie Fernsehen oder Zeitung und/oder private Kontakte auch von Formaten erfahren, die in ihrer Kita nicht angeboten werden, und bemängeln dieses.

«Ich hab bei Kollegen gefragt [...] und die haben Aufgabenpäckchen gekriegt von den Erziehern, da war ein direkter Draht per Videokonferenz jeden Morgen [...], dann haben die Erzieher eben ihr Morgenkreislied gemacht und kurze Interaktionen mit den Kindern und das hätte ich mir jetzt auch gewünscht.» (Vater eines Kindes, 45 Jahre)

Die vier Eltern, die den Bedarf an digitalen Kommunikationswegen mit den Kindern besonders hervorheben, erachten auch den pädagogischen Einsatz digitaler Medien in der Kita als sehr wichtig (siehe unten).

Mehrere Eltern äussern den Wunsch, von der Kita auch bezüglich des häuslichen Mediengebrauchs Unterstützung zu erhalten und dahingehend kompetente Fachkräfte als Ansprechpartnerinnen und -partner an ihrer Seite zu haben:

"[J]etzt zum Beispiel in der Coronazeit, gab es da ganz viel Tipps, was man zum Beispiel alles nutzen kann mit den Kindern, das fand ich wirklich wertvoll, weil ich sag mal, ich hab keine Zeit mich da überall reinzuarbeiten und zu suchen.» (Dreifache Mutter, 36 Jahre)

Die Kita sei ein guter Ort, um solche Tipps zu vermitteln, da - dies drücken mehrere Eltern aus - bereits ein Vertrauen in die Expertise der Fachkräfte und deren pädagogische Urteilskraft bestehe. 
«Also für mich wirkt dieser Gedanken eben auch entlastend, also dass ich so das Gefühl habe, okay, dann liegt diese Aufgabe eben nicht nur bei den Eltern.» (Mutter eines Kindes, 42 Jahre)

\section{Zu Annahme 2c (2)}

Der zweite Unterpunkt von Annahme $2 c$ besagt, dass die verstärkte digitale Ausrichtung der Kitas neben dem Wunsch nach mehr digitaler Kommunikation auch zu einer Verstärkung positiver Haltungen gegenüber Medienerziehung in der Kita geführt hat. Dies kann nur für die Haltungen von Eltern bestätigt werden, die dem Thema bereits vorher offen gegenüberstanden, kritisch-negative Haltungen blieben hingegen unverändert.

In der vollstandardisierten Onlinebefragung zeigt sich, dass die befragten Eltern gegenüber Medienerziehung in der Kita ähnlich eingestellt sind wie die Fachkräfte (siehe oben): Knapp die Hälfte gibt an, diese wichtig zu finden, nur jeder Zehnte befragte Person steht dem Thema skeptisch oder ablehnend gegenüber.

Auch in den Gruppendiskussionen wurden die Eltern nach ihrer Haltung zu Medienerziehung in der Kita gefragt. Hier hat sich ein heterogenes Bild mit Tendenz zu einer positiven Grundhaltung gezeigt. Auf einer Skala von 1 bis 10 ( $1=$ «digitale Medien gehören nicht in die Kita» bis $10=$ «digitale Medien gehören - wie analoge Medien auch - ganz normal in den Kita-Alltag»), haben sich sechs Eltern im Bereich 8-10, zwei im mittleren Bereich (4-7) und ein Elternteil mit einer kritisch-negativen Haltung bei 1 verortet $(M=7,78 ; S D=3,24)$. Erneut lässt sich die Tendenz erkennen, dass Eltern mit älteren Kindern ( $a b 3$ Jahren) eher eine positive Haltung und Eltern mit jüngeren Kindern (unter 2 Jahren) eher eine kritische Haltung vertreten (insbesondere, wenn das Kind keine älteren Geschwister hat).

Danach gefragt, ob durch den coronabedingten Lockdown eine Haltungsveränderung stattgefunden habe, betonen die meisten Eltern, dass der Lockdown und die damit verbundenen Erfahrungen keine Veränderung in den Haltungen hervorgebracht hätten. In drei Fällen allerdings geben die Eltern an, dass sich ihre ohnehin positive Haltung durch die besonderen Bedingungen noch verstärkt habe.

«[A]lso ich hatte die Haltung schon vorher, aber es hat halt noch mehr verdeutlicht, was für ein Potenzial dahintersteckt.» (Zweifacher Vater, 45 Jahre)

"[W]enn man sich darüber Gedanken macht, dann ist es eigentlich völlig klar, dass das sinnvoll ist. Also jetzt im Sinne von Medienpädagogik und Heranführung. Und ich mein, bei uns in der Kita ist da wirklich wenig in dem Bereich.» (Mutter eines Kindes, 42 Jahre) 
Diese verstärkte Sensibilisierung für das Thema wird sowohl durch gesellschaftspolitische Entwicklungen und den Digitalisierungsschub während der Coronapandemie sowie die damit salient gewordenen Potenziale digitaler Medien als auch durch eine verstärkte Auseinandersetzung mit dem Thema in Zusammenhang mit dem fortschreitenden Alter des Kindes erklärt.

«Das ist also irgendwie total paradox. Auf der einen Seite erkenne ich gerade, wie wichtig das ist, dass ich meine Prozesse umstelle, digitalisiere alles, aber die, die das später mal übernehmen sollen, die bleiben auf der Strecke.» (Zweifacher Vater, 45 Jahre)

\section{Diskussion und Fazit}

Die Coronapandemie hat den Kita-Alltag auf den Kopf gestellt - und den Raum für Veränderungen eingefahrener Prozesse geöffnet. Die Ergebnisse der vorliegenden Mixed-Methods-Studie, die in zehn Kindertageseinrichtungen durchgeführt wurde, zeigen, dass viele Veränderungen angestossen wurden, vor allem in Bezug auf den digitalen Kontakt zwischen den Kitas und Familien (Eltern bzw. Kindern). Entsprechend der Annahme 1a zeigt sich, dass Abläufe digitalisiert wurden, die vorher unhinterfragt analog stattfanden. Entsprechend der Annahme 1b konnten viele Fachkräfte ihre Fähigkeiten im Umgang mit digitalen Geräten ausbauen. Dies hat - gemäss Annahme $1 c$ - zu einem Abbau von Hemmungen bzw. Berührungsängsten geführt. Besonders eindrücklich scheinen die Erfahrungen dort zu sein, wo digitale Angebote klare Vorteile gegenüber ihren analogen Pendants hatten, d.h. etwa eine höhere Flexibilität boten oder mehr Eltern erreicht wurden. In mehreren Kitas gibt es im Kontext der vermehrten Praxiserfahrungen mit digitalen Medien im ersten Lockdown Anzeichen für eine offenere Grundhaltung des Teams. Hier stimmen die Daten mit denen von Cohen u. a. (2020) überein, welche zeigen, dass der Zuwachs an Erfahrungen auch die Haltungen der Fachkräfte verändert hat: So geben 41,6 \% der von ihnen befragten Kita-Fachkräfte an, sie stünden der Nutzung digitaler Medien für die Elternzusammenarbeit positiver gegenüber als vor der pandemiebedingten Schliesszeit, nur $1,7 \%$ sehen diese negativer.

Die im Rahmen der vorliegenden Studie durchgeführte quantitative Befragung hat gezeigt, dass unter den befragten Fachkräften das Wissen über die Bedeutung dieses Bildungsbereichs ungleich verteilt ist: Während einzelne Fachkräfte ein recht gutes Verständnis haben, ist dies nicht in der Mehrheit der Teams verankert. Die Ergebnisse zeigen jedoch auch, dass die - zumindest temporär - verstärkt digitale Ausrichtung der Kitas keineswegs sautomatisch) die langfristige und im Alltag verankerte pädagogische Auseinandersetzung mit digitalen Medien vorantreibt. Die Bandbreite der im ersten Lockdown getesteten Formate und ihr teilweise nur einmaliges Auftreten sprechen für eine gewisse Orientierungslosigkeit seitens der Kitas, was im 
Kontext der herausfordernden und neuartigen Situation des ersten Lockdowns nur wenig verwunderlich ist. Es entsteht der Eindruck, dass viele Entscheidungen eher ad hoc getroffen wurden und weniger auf eine Verstetigung ausgerichtet waren, denn als Reaktion auf akute Situationen. Aus dem weiteren Kontakt mit den befragten Einrichtungen ist allerdings erkennbar, dass die Auswahl der Kontaktwege im zweiten Lockdown gezielter erfolgt ist.

Hinsichtlich des während der Kita-Schliessungen angestiegenen häuslichen Einsatzes von Medien (siehe Annahme 2a) sind die Haltungen der Eltern gemischt und reichen von pragmatischen Ansätzen (‘Medieneinsatz hilft, den Alltag zu bewältigen und gibt den Eltern Freiräume zum Arbeiten im Homeoffice)) hin zu kritischen Haltungen, die auch mit Schuldgefühlen und Selbstvorwürfen verbunden sind (‘die Kinder werden vor den Geräten geparkt, weil es nicht anders machbar ist)). Während in vielen anderen gesellschaftlichen Bereichen die Veränderungen auch perspektivisch, d.h. über die Dauer der Pandemie hinaus betrachtet werden (etwa die Frage, ob auch weiterhin vermehrt im Homeoffice gearbeitet werden wird), scheinen die Veränderungen im Alltag der Kinder von den Eltern eher als kurzfristige Abweichungen gesehen zu werden, die stärker durch den Bedarf der Eltern denn durch Bedürfnisse der Kinder (nach sozialen Kontakten, Unterhaltung, Ablenkung, Beruhigung o. Ä.) erklärt werden. Es ist entgegen der Annahme $2 b$ nicht erkennbar, dass die vermehrte Mediennutzung von Kita-Kindern zu einer wesentlich höheren Sensibilität der Eltern gegenüber der Bedeutung von Medienerziehung geführt hat - wenn diese dem Thema nicht ohnehin bereits offen gegenüberstanden. Auch in Kitas, die bereits medienpädagogisch arbeiten, hat die überwiegende Mehrzahl der Eltern ein unklares oder undifferenziertes Verständnis davon, worauf dieser Bildungsbereich abzielt.

Unter den Eltern scheint es, als würden sie primär basierend auf ihren Alltagserfahrungen (die sich im Wesentlichen auf Regulierung und Einschränkung der kindlichen Mediennutzung beziehen) auf das Thema Medienerziehung blicken - diese Perspektive blieb auch im ersten Lockdown bzw. durch die digitalen Aktivitäten der Kita (siehe Annahme 2c) unverändert. Ihre Haltung gegenüber der Mediennutzung von Kindern (sowohl zu Hause als auch in der Kita) ist vom Alter des Kindes abhängig: Während Eltern mit kleineren Kindern (bis 3 Jahren) die Kita eher als Schonraum sehen und Medien im Familienalltag vermeiden, zeigen sich Eltern mit älteren Kindern (ab drei Jahren) offener.

In den Gruppendiskussionen mit Eltern hat sich bezüglich der Annahme $2 \mathrm{c}$ darüber hinaus gezeigt, dass die Vermittlung von Informationen via E-Mails, Videokonferenzen, Apps oder anderen digitalen Kommunikationswegen ob der höheren Praktikabilität und auch zeitlichen Flexibilität geschätzt wird (auch dieses Ergebnis deckt sich mit den Ergebnissen von Cohen u. a. 2020). Der digitale Kontakt zwischen Kita und den Kindern wird zwar überwiegend positiv gesehen, jedoch nicht von allen Eltern als wichtig erachtet. An Kinder adressierte und ins Digitale verlagerte 
pädagogische Angebote (etwa Morgenkreise o. Ä.) werden von den Eltern vor dem Hintergrund der Corona-Schliesszeiten als sinnvoll empfunden, jedoch kaum mit Forderungen nach einer über die Pandemie hinausgehenden Verstetigung einer gezielten Medienerziehung verknüpft.

Die hier vorgestellten Ergebnisse sind vor dem Hintergrund zu betrachten, dass sie in Kitas erhoben wurden, die an dem Forschungs- und Praxisprojekt «Medienerziehung im Dialog von Kita und Familie» teilnehmen, also über medienpädagogische Vorkenntnisse bzw. ein entsprechendes Interesse an der Thematik verfügen. So ist insbesondere die starke theoretische Beschäftigung der Kitas während des ersten Corona-Lockdowns mit medienpädagogischen Themen (Verankerung im Konzept, Anschaffung technischer Geräte, Planung von Schulungen etc.) auf diese Besonderheit des Samples zurückzuführen. Hingegen stellt die vermehrte digitale Kommunikation mit Kindern und Eltern keine Eigenart dieser zehn Einrichtungen dar, sondern wird ähnlich auch in anderen Erhebungen beobachtet (Schreyer und Lorenz 2020; Cohen u. a. 2020).

In Bezug auf die an den Gruppendiskussionen teilnehmenden Eltern ist limitierend hervorzuheben, dass diese eine ausgeprägte digitale Affinität vorweisen. Dies dürfte sich insbesondere auf die freiwillige Teilnahme an den Online-Gruppendiskussionen ausgewirkt haben, da die Verwendung eines Online-Videotools eine Hürde für Eltern mit niedriger Bedienkompetenz darstellt. Gruppendiskussionen in Präsenz waren jedoch aufgrund der coronabedingten Kontaktbeschränkungen nicht realisierbar. Diese Einschränkungen sind insbesondere hinsichtlich der durchschnittlich leicht positiven Tendenz der Eltern in Bezug auf frühkindliche Medienerziehung zu beachten. Auch die Teilnahme an den quantitativen Erhebungen war freiwillig. Hier haben sich prozentual wesentlich mehr Fachkräfte als Eltern beteiligt (gemessen an deren Gesamtzahl).

Entsprechend sind insbesondere die Ergebnisse aus der quantitativen Elternbefragung nur bedingt aussagekräftig für die Grundgesamtheit der Elternschaft. Wenngleich nicht abschliessend bewertet werden kann, inwiefern die Coronapandemie bei Fachkräften und Eltern ein breiteres Verständnis oder grössere Reflexion hinsichtlich der Bedeutung von Medienerziehung fördern konnte, zeigt sich, dass es durch Covid-19 und den Lockdown in den hier befragten Kitas diverse Anstösse hin zu einer offeneren und positiveren Grundhaltung gegenüber digitalen Medien gibt, die auf die Nutzung der neu erkannten Potenziale abzielen. Eingefahrene analoge Praktiken wurden in vielen Bereichen aufgebrochen, teilweise nutzten Fachkräfte auch freigewordene Ressourcen, um sich mit neuen Themen zu beschäftigen. Diese positiven Erfahrungen im beruflichen Alltag sind ein notwendiger erster Schritt, um nachhaltige Veränderungen hin zu einer breiten Verankerung von Medienpädagogik in Kindertagesstätten einzuleiten und beizubehalten. Sie sind jedoch keineswegs ein Selbstläufer, sondern erfordern anhaltende Bemühungen sowohl auf der praktischen Ebene 
der Kitas als auch vonseiten der Politik und der Träger bzw. organisatorischen Strukturen. Insbesondere ist eine klare Kommunikation sowohl gegenüber Fachkräften als auch Eltern bezüglich der Inhalte und Ziele des Bildungsbereichs Medienerziehung notwendig, da hier grosse Unklarheiten persistieren. Die Covid-19-Pandemie bietet in diesem Zusammenhang einen relevanten Ausgangspunkt, um sowohl Fachkräfte als auch Eltern für die Bedeutung digitaler Medien im Alltag von Kita-Kindern zu sensibilisieren - und entsprechend auch für eine pädagogisch begleitete Heranführung.

Wie in vielen anderen gesellschaftlichen Bereichen kann sich die Pandemie auch in der frühkindlichen Medienerziehung als Gamechanger erweisen, wenn die beschriebenen Ansätze verstetigt und im Sinne eines nachhaltigen, über die Anforderungen der Pandemie hinausreichenden Wandels genutzt werden. Hierfür ist es wichtig, dass im gesellschaftlichen Fachdiskurs die Bedeutung digitaler Medien in Kitas nicht nur auf die Kommunikation zu den Eltern und Kindern (z. B. während der Schliesszeiten) beschränkt wird. Vielmehr sollte die Bedeutung digitaler Medien im Leben der Kinder und das damit einhergehende Erfordernis, diese auch über die Pandemie hinaus im pädagogischen Alltag aufzugreifen, explizit hervorgehoben werden.

\section{Literatur}

Andresen, Sabine, Anna Lips, Renate Möller, Tanja Rusack, Wolfgang Schröer, Severine Thomas, und Johanna Wilmes. 2020a. «Erfahrungen und Perspektiven von jungen Menschen während der Corona-Maßnahmen». Hildesheim: Universitätsverlag Hildesheim. https:// doi.org/10.18442/120.

Andresen, Sabine, Anna Lips, Renate Möller, Tanja Rusack, Wolfgang Schröer, Severine Thomas, und Johanna Wilmes. 2020b. "Kinder, Eltern und ihre Erfahrungen während der Corona-Pandemie». Hildesheim: Universitätsverlag Hildesheim. https://doi.org/10.18442/121.

Anfang, Günther, und Katrin Demmler. 2018. «Medienkompetenzförderung in der Kita». merz medien + erziehung., Kita digital: Frühe Medienerziehung, 2018 (2): 12-20.

Anger, Silke, Sarah Bernhard, Hans Dietrich, Adrian Lerche, Alexander Patzina, Malte Sandner, und Carina Toussaint. 2020. «Schulschließungen wegen Corona: Regelmäßiger Kontakt zur Schule kann die schulischen Aktivitäten der Jugendlichen erhöhen». IAB-Forum 23. April 2020, 11. https://www.iab-forum.de/schulschliessungen-wegen-corona-regelmassigerkontakt-zur-schule-kann-die-schulischen-aktivitaten-der-jugendlichen-erhohen/.

Baacke, Dieter. 2007. Medienpädagogik. Nachdr. Grundlagen der Medienkommunikation 1. Tübingen: Niemeyer. 
Bartels, Wolfgang. 2020. «Berlin schaut auf Hüffelsheimer Vorzeigeprojekt». Allgemeine Zeitung, 26. Oktober 2020. https://www.allgemeine-zeitung.de/lokales/bad-kreuznach/vgruedesheim/hueffelsheim/berlin-schaut-auf-huffelsheimer-vorzeigeprojekt_22486292.

Blom, Annelies, Alexander Wenz, Tobias Rettig, Maximiliane Reifenscheid, Elias Naumann, Katja Möhring, Roni Lehrer, u. a. 2020. «Die Mannheimer Corona-Studie: Das Leben in Deutschland im Ausnahmezustand. Bericht zur Lage vom 20. März bis 09. Juli 2020.» Mannheim: Universität Mannheim. https://www.uni-mannheim.de/media/Einrichtungen/gip/ Corona_Studie/10-07-2020_Mannheimer_Corona-Studie_-_Bericht_zur_Lage_in_den_Tagen_20_Mrz-09_Jul_2020.pdf.

Bujard, Martin, Inga Laß, Sabine Diabaté, Harun Sulak, und Norbert F. Schneider. 2020. «Eltern während der Corona-Krise. Zur Improvisation gezwungen». Herausgegeben von Bundesinstitut für Bevölkerungsforschung, BiB.Bevölkerungs.Studien, 2020 (1). https://doi. org/10.12765/bro-2020-0.

Bundesministerium für Familie, Senioren, Frauen und Jugend. 2020. «Familien in der Corona-Zeit: Herausforderungen, Erfahrungen und Bedarfe. Ergebnisse einer repräsentativen Elternbefragung im April und Mai 2020». Berlin. https://www.bmfsfj.de/resource/blob/16 3126/62d9765b8c54986e69d0ed0eb69f21df/familien-in-der-corona-zeit-elternbefragung2020-data.pdf.

Bundesministerium für Familie, Senioren, Frauen und Jugend [@BMFSFJ]. 2021. «Statement von Franziska \#Giffey zum heutigen \#CoronaKiTaRat mit Ländern, Kommunen, \#KiTa-Trägern, Gewerkschaften, Bundesverband für \#Kindertagespflege, Bundeselternvertretung sowie Kinder- und JugendärztInnen.» Tweet. Twitter. https://twitter.com/BMFSFJ/status/1346106388750032898.

Cohen, Franziska, Elisa Oppermann, und Yvonne Anders. 2020. «Familien \& Kitas in der Corona-Zeit. Zusammenfassung der Ergebnisse». Bamberg: Otto-Friedrich-Universität Bamberg. https://www.uni-bamberg.de/fileadmin/efp/forschung/Corona/Ergebnisbericht_finale_Version_Onlineversion.pdf.

Damm, Paul. 2020. «Digitaler Morgenkreis in Wittenberg Kindergarten vor der Flimmerkiste». Mitteldeutsche Zeitung, 6. April 2020. https://www.mz-web.de/wittenberg/digitaler-morgenkreis-in-wittenberg-kindergarten-vor-der-flimmerkiste-36518996.

Deutscher Caritasverband. 2020. "Corona-Kita-Rat: Kitas stärken Eltern, dafür brauchen sie eine bessere digitale Ausstattung». Pressemitteilung. https://www.caritas-regensburg.de/ pressemitteilungen/corona-kita-rat-kitas-staerken-eltern-dafuer-brauc/1769518/.

Deutsches Jugendinstitut und Robert-Koch-Institut. 2020. «Corona-Kita-Studie». https://www. corona-kita-studie.de.

Dresing, Thorsten, und Thorsten Pehl. 2018. Praxisbuch Interview, Transkription \& Analyse. Anleitungen und Regelsysteme für qualitativ Forschende. 8. Aufl. Marburg. https://www.audiotranskription.de/wp-content/uploads/2020/11/Praxisbuch_08_01_web.pdf. 
Eckert, Marion. 2020. «Unterelsbach: In Corona-Zeiten wird auch der Kindergarten digital». Mainpost, 3. April 2020. https://www.mainpost.de/regional/rhoengrabfeld/unterelsbachin-corona-zeiten-wird-auch-der-kindergarten-digital-art-10430961.

Eder, Sabine. 2018. «Die frühe Medienpädagogik steht vor großen Herausforderungen. Ein Interview mit Sabine Eder, Blickwechsel e.V.» merz medien + erziehung, Kita digital: Frühe Medienerziehung, 2018 (2): 49-53.

Eder, Sabine, Anke Lehmann, André Lenich, Susanne Roboom, Gerhard Seiler, und Johannis Wentzel. 2013. Medienkompetenz-Kitas NRW: ein Modellprojekt der Landesanstalt für Medien Nordrhein-Westfalen (LFM). Herausgegeben von Landesanstalt für Medien NordrheinWestfalen. Düsseldorf: LfM. https://www.medienanstalt-nrw.de/fileadmin/user_upload/ materials_and_ordering_system/download/L155_Medienkompetenz-Kitas-NRW.pdf.

Eggert, Susanne, und Ulrike Wagner. 2016. "Grundlagen zur Medienerziehung in der Familie. Expertise im Rahmen der Studie MoFam - Mobile Medien in der Familie.» JFF - Institut für Medienpädagogik in Forschung und Praxis. https://doi.org/10.25656/01:16560.

Entringer, Theresa, und Magdalena Krieger. 2020. «Spotlight 3 der SOEP-CoV Studie: Alleinlebende verkraften die Pandemie erstaunlich gut». SOEP-CoV - Sozio-ökonomische Faktoren und Folgen der Verbreitung des Coronavirus in Deutschland. https://www.soep-cov.de/. cm4all/uproc.php/0/SOEP_CoV_Spotlight_3.pdf.

Feierabend, Sabine, Theresa Plankenhorn, und Thomas Rathgeb. 2015. «miniKIM 2014. Kleinkinder und Medien. Basisuntersuchung zum Medienumgang 2-5-Jähriger in Deutschland». Stuttgart. https://www.mpfs.de/fileadmin/files/Studien/miniKIM/2014/Studie/miniKIM_ Studie_2014.pdf.

Fleischer, Sandra, Peter Kroker, und Susanne Schneider. 2018. «Medien, frühe Kindheit und Familie». In Frühe Kindheit und Medien - Aspekte der Medienkompetenzförderung in der Kita, herausgegeben von Jürgen Georg Brandt, Manfred Kaulbach, Christine Hoffmann, und Thomas Schmidt, 35-52. Opladen Berlin Toronto: Verlag Barbara Budrich. https://doi. org/10.1007/978-3-531-18997-0_23.

Friedrichs-Liesenkötter, Henrike. 2016. Medienerziehung in Kindertagesstätten: Habitusformationen angehender ErzieherInnen. Medienbildung und Gesellschaft, Band 34. Wiesbaden: Springer VS. https://doi.org/10.1007/978-3-658-12307-9.

Friedrichs-Liesenkötter, Henrike. 2018. «Und das Handy hat sie von der Zahnfee gekriegt! Medienerziehung in Kindertagesstätten unter dem Blickwinkel des medienerzieherischen Habitus angehender Erzieher/innen». In Frühe Kindheit und Medien - Aspekte der Medienkompetenzförderung in der Kita, herausgegeben von Jürgen Georg Brandt, Christine Hoffmann, Manfred Kaulbach, und Thomas Schmidt, 53-76. Opladen Berlin Toronto: Verlag Barbara Budrich. https://doi.org/10.1007/978-3-531-18997-0_23.

Friedrichs-Liesenkötter, Henrike. 2019. «Wo Medienbildung draufsteht, steckt nicht unbedingt Medienbildung drin> Eine Dokumentenanalyse von Bildungsplänen und Curricula in Ausbildung und Studium zur frühkindlichen Medienbildung und -erziehung». Medienimpulse 57 (1). https://doi.org/10.21243/mi-01-19-07. 
Fröbel e.V. 2020. «Wie in der Coronakrise aus Risiken Chancen werden». Pressemitteilung. https://www.froebel-gruppe.de/aktuelles/news-single/artikel/wie-in-der-coronakrise-ausrisiken-chancen-werden/.

Geis-Thöne, Wido. 2020. «IW-Report 15/2020. Häusliches Umfeld in der Krise: Ein Teil der Kinder braucht mehr Unterstützung». Köln. https://www.iwkoeln.de/fileadmin/user_upload/ Studien/Report/PDF/2020/IW-Report_2020_Haeusliche_Lebenswelten_Kinder.pdf.

Götz, Maya, Caroline Mendel, Dafna Lemish, Nancy Jennings, Rebecca Hains, Fatima Abdul, Meryl Alper, u. a. 2020. «Children, COVID-19 and the Media». Televizion, Nr. 33: 4-9. http:// www.br-online.de/jugend/izi/english/publication/televizion/33_2020_E/Goetz_Mendel_ Lemish-Children_COVID-19_and_the_media.pdf.

Grobbin, Alexander. 2016. Digitale Medien: Beratungs-, Handlungs- und Regulierungsbedarf aus Elternperspektive. Abschlussbericht. Herausgegeben von Deutsches Jugendinstitut. München. http://nbn-resolving.de/urn:nbn:de:101:1-201610254110.

Grüling, Birk. 2020. «Briefe aus der Kita». DIE ZEIT, 29. April 2020. https://www.zeit.de/gesellschaft/2020-04/paedagogie-coronavirus-kitaschliessung-kontaktstopp-kinder-bindung.

Guth, Birgit. 2018. «Vorschulkinder im Fokus. Mediennutzung bei den Kleinen». Kinderwelten.

Hajok, Daniel. 2020. «Heranwachsende im Ausnahmezustand: Homeschooling, verändertes Zusammenleben und Medienumgang in Coronazeiten». tv Diskurs 2020 (3): 10-13. https:// tvdiskurs.de/data/hefte/ausgabe/93/hajok-heranwachsende-corona-tvd93.pdf.

Heidland, Kerstin. 2020. «Duisburger Kirchengemeinde feiert digitales St. Martinsfest». WAZ, 5. November 2020. https://www.waz.de/staedte/duisburg/west/duisburger-kirchengemeinde-feiert-digitales-st-martinsfest-id230844954.html.

Heundorf, Julia. 2020. «Notbetreuung in Magdeburg: Wie digitale Kita funktioniert». MDR Sachsen-Anhalt, 21. März 2020. https://www.mdr.de/sachsen-anhalt/magdeburg/magdeburg/ digitale-kita-mandala-100.html.

Huebener, Mathias, C. Katharina Spieß, und Sabine Zinn. 2020. «SchülerInnen in Corona-Zeiten: Teils deutliche Unterschiede im Zugang zu Lernmaterial nach Schultypen und -trägern». DIW Wochenbericht 2020 (47). https://doi.org/10.18723/DIW_WB:2020-47-1.

Institut für Demoskopie Allensbach. 2014. «Digitale Medienbildung in Grundschule und Kindergarten. Ergebnisse einer Befragung von Eltern, Lehrkräften an Grundschulen und Erzieher(innen) in Kindergärten im Auftrag der Deutsche Telekom Stiftung». https://www. telekom-stiftung.de/sites/default/files/files/media/publications/ergebnisse_allensbachumfrage_gesamt.pdf.

Langmeyer, Alexandra, Angelika Guglhör-Rudan, Thorsten Naab, Marc Urlen, und Ursula Winklhofer. 2020. «Kindsein in Zeiten von Corona. Erste Ergebnisse zum veränderten Alltag und zum Wohlbefinden von Kindern». Herausgegeben von Deutsches Jugendinstitut. Deutsches Jugendinstitut. https://www.dji.de/fileadmin/user_upload/dasdji/themen/Familie/ DJI_Kindsein_Corona_Erste_Ergebnisse.pdf. 
Liebig, Stefan. 2020. «Spotlight 2 der SOEP-CoV Studie: Familienleben im Lockdown». SOEPCoV - Sozio-ökonomische Faktoren und Folgen der Verbreitung des Coronavirus in Deutschland. https://www.soep-cov.de/.cm4all/uproc.php/0/SOEP_CoV_Spotlight_2.pdf.

Lienau, Theresa, und Lies van Roessel. 2019. «Zur Verankerung von Medienerziehung in den Bildungsplänen für Kindertageseinrichtungen». MedienPädagogik: Zeitschrift für Theorie und Praxis der Medienbildung 2019 (Occasional Papers): 126-55. https://doi.org/10.21240/ mpaed/00/2019.12.01.X.

PM. 2020. «Wenn das Kind nicht in die Kita kommt, kommt die Kita zum Kind». AK-Kurier, 28. Mai 2020. https://www.ak-kurier.de/akkurier/www/artikel/90745-wenn-das-kind-nicht-indie-kita-kommt--kommt-die-kita-zum-kind.

Rathgeb, Thomas. 2020. «JIMplus 2020: Lernen und Freizeit in der Corona-Krise». Medienpädagogischer Forschungsverbund Südwest. https://www.mpfs.de/fileadmin/files/Studien/ JIM/JIMplus_2020/JIMplus_2020_Corona.pdf.

Rosebrock, Cornelia, und Olga Zitzelsberger. 2002. «Der Begriff Medienkompetenz als Zielperspektive im Diskurs der Pädagogik und Didaktik». In Medienkompetenz: Voraussetzungen, Dimensionen, Funktionen, herausgegeben von Norbert Groeben und Bettina Hurrelmann, 148-59. Weinheim [u.a.]: Juventa.

Schreyer, Inge, und Sigrid Lorenz. 2020. «Zoom - 4. Newsletter für das Projekt „Medienkompetenz in der Frühpädagogik stärken“». https://www.kita-digital-bayern.de/files/media/ public/downloads/einblicke-und-ergebnisse/ZOOM-Newsletter-Nr-4-September-2020.pdf.

Schubert, Gisela, Niels Brüggen, Andreas Oberlinner, Susanne Eggert, und Valerie Jochim. 2018. «Haltungen von pädagogischem Personal zu mobilen Medien, Internet und digitalen Spielen in Kindertageseinrichtungen. Bericht der Teilstudie „Mobile Medien und Internet im Kindesalter - Fokus Kindertageseinrichtungen“». München. https://doi. org/10.25656/01:18042.

Schubert, Gisela, Susanne Eggert, Anne Lohr, Andreas Oberlinner, Valerie Jochim, und Niels Brüggen. 2018. «Digitale Medien in Kindertageseinrichtungen: Medienerzieherisches Handeln und Erziehungspartnerschaft Perspektiven des pädagogischen Personals. Zweiter Bericht der Teilstudie „Mobile Medien und Internet im Kindesalter - Fokus Kindertageseinrichtungen» im Rahmen von MoFam - Mobile Medien in der Familie». München: JFF Institut für Medienpädagogik in Forschung und Praxis. https://doi.org/10.25656/01:1608.

Six, Ulrike, Christoph Frey, und Roland Gimmler. 1998. Medienerziehung im Kindergarten: theoretische Grundlagen und empirische Befunde. Schriftenreihe Medienforschung der Landesanstalt für Rundfunk Nordrhein-Westfalen, Bd. 28. Opladen: Leske + Budrich.

Six, Ulrike, und Roland Gimmler. 2007. Die Förderung von Medienkompetenz im Kindergarten: eine empirische Studie zu Bedingungen und Handlungsformen der Medienerziehung. Schriftenreihe Medienforschung der Landesanstalt für Medien Nordrhein-Westfalen 57. Berlin: vistas. 
Stiftung Haus der kleinen Forscher. 2017. "„Wie nutzen Erzieherinnen und Erzieher digitale Geräte in Kitas?" - Eine repräsentative Telefonumfrage». Berlin. https://www.haus-derkleinen-forscher.de/fileadmin/Redaktion/3_Aktuelles/Presse/171213_Ergebnisse_zur_Telefonbefragung_Digitales.pdf.

Theunert, Helga, und Kathrin Demmler. 2007. «(Interaktive) Medien im Leben Null- bis Sechsjähriger - Realitäten und Handlungsnotwendigkeiten». In Digitale Medien in der Schule. Standortbestimmung und Handlungsempfehlungen für die Zukunft. Studie zur Nutzung digitaler Medien in allgemein bildendenden Schulen in Deutschland., herausgegeben von Bardo Herzig und Silke Grafe, Digitale Medien in der Schule. Standortbestimmung und Handlungsempfehlungen für die Zukunft. Studie zur Nutzung digitaler Medien in allgemein bildendenden Schulen in Deutschland.:137-45. Bonn: Deutsche Telekom.

Tulodziecki, Gerhard. 2008. «Medienerziehung». In Handbuch Medienpädagogik, herausgegeben von Uwe Sander, Friederike von Gross, und Kai-Uwe Hugger, 110-15. Wiesbaden: VS Verlag für Sozialwissenschaften. https://doi.org/10.1007/978-3-531-91158-8_12.

Zinn, Sabine. 2020. «Spotlight 1 der SOEP-CoV Studie: Familienleben in Corona-Zeiten». SOEP-CoV - Sozio-ökonomische Faktoren und Folgen der Verbreitung des Coronavirus in Deutschland.

Zinn, Sabine, Michael Bayer, Theresa Entringer, Jan Goebel, Markus M Grabka, Daniel Graeber, Martin Kroh, u. a. 2020. «Subjektive Belastung der Eltern durch Schulschließungen zu Zeiten des Corona-bedingten Lockdowns». Herausgegeben von Deutsches Institut für Wirtschaftsforschung (DIW). SOEPpapers 2020 (1097): 20. http://hdl.handle.net/10419/222656. 